\title{
Influence of Lane Policies on Freeway Traffic Mixed with Manual and Connected and Autonomous Vehicles
}

\author{
Xuedong Hua $\mathbb{D}^{1,2,3}$ Weijie Yu $\mathbb{D}^{1,2,3}$ Wei Wang $\left.\mathbb{D}\right)^{1,2,3}$ and Wenjie Xie $\mathbb{D}^{1,2,3}$ \\ ${ }^{1}$ Jiangsu Key Laboratory of Urban ITS, Southeast University, Nanjing 211189, China \\ ${ }^{2}$ Jiangsu Province Collaborative Innovation Center of Modern Urban Traffic Technologies, Southeast University, \\ Nanjing 211189, China \\ ${ }^{3}$ School of Transportation, Southeast University, Nanjing 211189, China \\ Correspondence should be addressed to Xuedong Hua; qdurgk@163.com
}

Received 16 August 2019; Accepted 21 January 2020; Published 20 February 2020

Academic Editor: Filomena Mauriello

Copyright ( 2020 Xuedong Hua et al. This is an open access article distributed under the Creative Commons Attribution License, which permits unrestricted use, distribution, and reproduction in any medium, provided the original work is properly cited.

\begin{abstract}
Connected and autonomous vehicles (CAVs) have become the highlights of traffic. Researchers in this field have proposed various traffic management measures to enhance the capacity and efficiency of traffic with CAVs, especially mixed traffic of CAVs and manual vehicles (MVs). Exclusive lane setting is included. However, exclusive lane policy-related researches for mixed traffic of CAVs and MVs were very limited, and the influence of number and location of exclusive lanes on the mixed traffic was unclear. To fill this gap, this paper aims to study the influence of different exclusive lane policies on mixed traffic and provide recommended lane policies under various traffic volumes and CAV penetration rates. Freeways with two lanes and three lanes in a single direction were taken into consideration, and sixteen lane policies were proposed. Then different lane policies were simulated with a new proposed cellular automata (CA) model, and properties including flux, average speed, and CAVs degradation were analyzed to evaluate the traffic efficiency of each lane policy. The results show that CAV exclusive lanes can improve the capacity, while MV exclusive lanes seem helpless for capacity improvement. Seven lane policies, including GC, GM, and CM for two-lane freeways and GCG, CGC, and CCM for three-lane freeways, outperform the others in terms of average speed. In addition, exclusive lanes can reduce the probability that CAVs degenerate to AVs. Our findings may help to optimize freeways' lane policies and improve the efficiency of heterogeneous traffic mixed with CAVs and MVs.
\end{abstract}

\section{Introduction}

In the past decades, scholars and engineers have been seeking solutions to the questions of how the traffic capacity could be enhanced more efficiently and how traffic operations can be improved by intelligent transportation systems (ITS) [1]. Developments in artificial intelligence, sensor technology, and communication technology have led to significant advancements in ITS. In this context, connected and autonomous vehicles (CAVs) are now coming to the fore [2].

CAVs have become the highlights and attracted worldwide attention because of superior characteristics such as driverless navigation and vehicle-to-vehicle (V2V) and vehicle-to-infrastructure (V2I) communication abilities
$[3,4]$. Compared with human drivers, CAVs can get more accurate traffic information in nearly real-time, thus making it possible for CAVs to instantly react to the changes in driving conditions without delay [5]. The headway between two successive CAVs is quite smaller than that between two manual vehicles (MVs) [6-8]. Further, V2V and V2I greatly enlarge the detection range of CAVs' sensors, which means an ability to obtain traffic information from hundreds of meters ahead and in advance.

However, the mixed traffic of CAVs and MVs is bound to exist for a long period. Traffic operations will be affected by the interaction between CAVs and MVs $[9,10]$. This mixed traffic is unavoidable and can result in efficiency reduction and safety issues when compared with pure CAV traffic. Traffic management approaches including exclusive lane 
settings to relieve the interaction and to improve the performance of mixed traffic are worthy to be studied. This paper developed a cellular automata (CA) model to describe the different vehicular driving behaviors of CAVs and MVs. The influence of different exclusive lane policies on mixed traffic consisting of CAVs and MVs under various traffic volumes and CAV penetration rates $\left(P_{\mathrm{CAV}}\right)$ was carefully investigated. At last, recommendations for the exclusive lane setup were drawn.

This paper is organized as follows. First, a review of relevant literature is presented in Section 2. A general microscope traffic model that can describe both CAVs and MVs is proposed in Section 3. Section 4 introduces the setup of different exclusive lane policies. Section 5 analyses the simulation results. The influence of some importation factors on mixed traffic and lane policies is investigated in Section 6. Section 7 draws the conclusions and outlooks future research.

\section{Literature Review}

Previously, the influences of autonomous driving and V2V communication on traffic, e.g., influence on traffic safety, capacity, congestion, and behaviors, have been studied. For example, Daniel [11] investigated the potential benefits from autonomous vehicles (AVs) to reduce crashes, ease congestion, improve fuel economy, and reduce parking needs. The significant barriers to full implementation which AVs faced were considered as well. Mahmassani [12] focused on AVs' and connected vehicles' (CVs) impact on traffic flow and operations. He found that, with an increased market share, AVs exerted a greater influence on both dimensions compared with the same shares of CVs.

Studies concerning both autonomous and connected vehicles were conducted since the 1990s [13-15], to reveal the traffic flow properties under mixed traffic conditions. Traffic capacity, affected by vehicle composition, was widely investigated. Ghiasi et al. [16] developed a CAV-based trajectory-smoothing algorithm to harmonize traffic and improve mobility. The results showed significant improvement in traffic capacity and fuel consumption. Talebpour and Mahmassani [17] presented a framework to simulate different CAVs with distinct communication capabilities and showed that CAVs could improve road capacity by more than $100 \%$. In these studies, the impact of CAVs on road capacity increase was substantial under certain market penetration scenarios. Hence, a large number of studies tried to find the relationship between capacity growth and CAV penetration rate $[1,2,18,19]$. Besides, the increase of road capacity indicates a smaller average headway, which may bring safety issues. To deal with this, studies were carried out to find a balance between increases of traffic capacity and accident risks [20, 21].

Except for capacity, the traffic efficiency of the heterogeneous traffic was discussed. CAVs proved to be beneficial for average speed improvement and travel time reduction [22]. Learn et al. [23] developed an innovative vehicle control platform and designed an experiment to conduct speed harmonization field experiments. The experiment results showed the potential of CAVs in reducing the oscillation of traffic. Ye and Yamamoto [2] proposed a simulation method wherein both the CAVs and MVs were incorporated. The results showed that the average speed increased with an increased CAV penetration rate when the density was over about $30 \mathrm{veh} / \mathrm{km}$.

In addition, exclusive lanes, a traffic management approach, were widely applied to solve the traffic chaos brought by mixed traffic. The implementation of exclusive lanes such as bus lanes and high-occupancy vehicle (HOV) lanes was beneficial [24-26]. Learning from the previous successful experience, the CAV-exclusive lane is a feasible approach to reduce the interaction between CAVs and MVs and to improve the performance of mixed traffic [27-29]. Ma and Wang [30] developed a four-lane heterogeneous CA flow model and studied the impact of exclusive lanes for CAVs on the overall flow throughput. His study revealed that the traffic volume of roads with the exclusive lane for CAVs, in most cases, exceeds the operation result of roads without exclusive lanes. Mahmassani [12] examined the effectiveness of exclusive lanes and illustrated that the performance of CAV lanes was good only if when the market share of CAVs was greater than the percentage of nominal capacity represented by that lane. Ghiasi et al. [9] built a lane management model to efficiently determine the optimal number of exclusive CAV lanes to maximize mixed traffic throughput.

Based on the review of related literature about the impact of CAVs on traffic flow and operations, some limits can be observed. Although CAV exclusive lanes were demonstrated, MV exclusive lanes, however, were neglected in the previous studies. The impact of MV exclusive lanes, as well as a combination of CAV exclusive lanes and MV exclusive lanes, on traffic flow, is not clear. Additionally, the influence of the number and the location of exclusive lanes on the mixed traffic needs to be further analyzed. In this study, a freeway segment with three lanes in a single direction was taken into consideration, and twelve exclusive lane policies were then proposed and compared. A CA simulation model was developed to evaluate the influence of lane policies on mixed traffic. Further, how factors (e.g., headway values) affect the performance of exclusive lane policies was analyzed and discussed as well.

\section{Modeling}

The CA model is one of the most widely applied microscopic simulation models [31]. Although the accuracy is a bit lower compared with other continuous models, advantages such as simplicity of modeling process, good capability of describing driving behaviors, and real traffic characteristics have been demonstrated in the past researches [30]. Some typical CA models including the KKW model [32], MCD model [33], and TS model [34] have been proposed. CA models after modification are suitable for sophisticated traffic composed of MVs and CAVs [1]. The TS model can well reproduce the metastable state, traffic oscillations, phase transitions, and other real traffic flow dynamics [2]. Besides, compared with other CA models, TS model performs the best [34]. So, in this paper, a TS model-based heterogeneous flow model is adopted, which takes the influence of communication, 
autonomous driving, and human driving behaviors into account.

3.1. Notion of Model Parameters and Variables. To facilitate the CA model setup, the notation used in this paper is summarized in Table 1.

3.2. Car-Following Submodel for MVs. For car-following process of MVs, three steps are involved, as follows:

Step 1 (deterministic speed update).

The deterministic speed, $v_{\mathrm{det}}^{\mathrm{MV}}$, is determined by the minimum value of $v+a, v_{\max }, d_{\text {anti }}$ and $v_{\text {safe }}$, as shown in the following equation:

$$
v_{\mathrm{det}}^{\mathrm{MV}}=\min \left(v+a, v_{\max }, d_{\mathrm{anti}}, v_{\mathrm{safe}}\right) .
$$

where $d_{\text {anti }}$ helps to keep a safe distance if the preceding vehicle travels at an expected velocity, while $v_{\text {safe }}$ helps in avoiding accidents in case of preceding vehicle's sudden braking and $d_{\text {anti }}$ and $v_{\text {safe }}$ are demonstrated in the following equations, respectively:

$$
\begin{aligned}
& d_{\mathrm{anti}}=\min \left(\frac{d+v_{\mathrm{anti}}+L_{\mathrm{veh}}}{1+g_{\text {safety }}}, d+v_{\mathrm{anti}}\right), \\
& v_{\mathrm{safe}}=\left[-b_{\max }+\sqrt{b_{\text {max }}^{2}+v_{l}^{2}+2 b_{\max } d}\right],
\end{aligned}
$$

where $g_{\text {safety }}$ equals to $g_{\mathrm{MV}-\mathrm{MV}}$ if the preceding vehicle is an $\mathrm{MV}$, or equals to $g_{\mathrm{MV}-\mathrm{CAV}}$ if the preceding vehicle is a CAV. Function [.] in equation (3) can return a nearest integer. $v_{\text {anti }}$ is defined as equation (4). Note that, equation (3) is derived from Gipps' model [35] with the human reaction time set as $1 \mathrm{~s}:$

$$
v_{\text {anti }}=\min \left(d_{l}, v_{l}+a, v_{\max }\right)
$$

Step 2 (stochastic deceleration).

MVs may be deceleration stochastically, which can be described as follows:

$$
v^{\prime}= \begin{cases}\max \left(v_{\mathrm{det}}^{\mathrm{MV}}-b_{\text {rand }}, 0\right), & \text { with probability } p, \\ v_{\mathrm{det}}^{\mathrm{MV}}, & \text { otherwise. }\end{cases}
$$

The randomization deceleration value $b_{\text {rand }}$ and probability $p$ are defined as follows:

$$
\begin{gathered}
b_{\text {rand }}= \begin{cases}a, & \text { if } v \leq b_{\text {defense }}+\left\lfloor\frac{d_{\text {anti }}}{T}\right\rfloor, \\
b_{\text {defense }}, & \text { otherwise, }\end{cases} \\
p= \begin{cases}p_{b}, & \text { if } v=0, \\
p_{c}, & \text { else if } v \leq\left(\frac{d_{\text {anti }}}{T}\right), \\
p_{\text {defence, }}, & \text { otherwise, }\end{cases}
\end{gathered}
$$

where $p_{\text {defence }}=p_{c}+\left(p_{a} / 1+e^{\alpha\left(v_{c}-v\right)}\right)$.

Step 3 (position update).

The location of a MV can be obtained by the following equation:

$$
x^{\prime}=x+v^{\prime}
$$

3.3. Car-Following Submodel for CAVs. Compared with MVs, CAVs will not decelerate stochastically. In the CAV car-following submodel, the stochastic deceleration step is neglected, and only two steps are involved.

Step 1 (deterministic speed update).

$$
v_{\mathrm{det}}^{\mathrm{CAV}}=\min \left(v+a_{\mathrm{CAV}}, v_{\mathrm{max}}, d_{\mathrm{anti}}, v_{\mathrm{safe}}\right) .
$$

The acceleration of CAV, $a_{\mathrm{CAV}}$, can be determined by a typical ACC model proposed in [36], as shown in the following equations:

$$
\begin{aligned}
a & =K_{1}\left(d-v T_{\text {desired }}\right)+K_{2}\left(v_{l}-v\right), \\
-b_{\max } & \leq a \leq a_{\max } .
\end{aligned}
$$

The anticipated space gap for CAV can be calculated by equation (2) as well. The $g_{\text {safety }}$ for CAVs is different from that for MVs. When the preceding vehicle is $\mathrm{MV}, g_{\text {safety }}$ equals to $g_{\mathrm{CAV}-\mathrm{MV}}$; otherwise, it equals to $g_{\mathrm{CAV}-\mathrm{CAV}}$.

As CAV can obtain information by communication, the calculation of the expected velocity of the preceding vehicle will be a bit different: if the preceding vehicle is a CAV, it can be defined as equation (11); otherwise, equation (4) is adopted:

$$
v_{\mathrm{anti}}=\min \left(d_{l}, v_{l}+a_{l}, v_{\max }, \bar{v}_{l}\right)
$$

The safe speed is calculated by equation (12), which is similar to equation (3). In equation (12), the $1 \mathrm{~s}$ human reaction time is neglected and the communication delay, $\Delta t$, is considered:

$$
v_{\text {safe }}=\left[-b_{\max } \Delta t+\sqrt{b_{\max }^{2} \Delta t^{2}+v_{l}^{2}+2 b_{\max } d}\right] .
$$

Step 2 (position update).

$$
x^{\prime}=x+v_{\mathrm{det}}^{\mathrm{MV}} .
$$

3.4. Lane-Changing Submodel. When the following conditions are all fulfilled, MVs will move onto the target lane with a probability $p_{l c}$ :

$$
\begin{aligned}
& d<\min \left(v+1, v_{\max }\right), \\
& d_{\text {other }}>d, \\
& d_{\text {other }}^{\text {back }} \geq v_{\max } .
\end{aligned}
$$


TABLE 1: Notation of model parameters and variables.

\begin{tabular}{|c|c|c|}
\hline Notion & Explanation & Unit \\
\hline$L_{\text {cell }}$ & Length of one cell & $\mathrm{m}$ \\
\hline$v_{\mathrm{det}}^{\mathrm{MV}}$ & Deterministic speed of MV at subsequent time step & $L_{\text {cell }} / \mathrm{s}$ \\
\hline v' & Speed of MV at subsequent time step after stochastic deceleration & $L_{\text {cell }} / \mathrm{s}$ \\
\hline$v$ & The speed at the current time step & $L_{\text {cell }} / \mathrm{s}$ \\
\hline$a$ & Acceleration rate & $L_{\text {cell }} / \mathrm{s}^{2}$ \\
\hline$v_{\max }$ & Maximum speed & $L_{\text {cell }} / \mathrm{s}$ \\
\hline$d_{\text {anti }}$ & Anticipated space gap & $L_{\text {cell }}$ \\
\hline$v_{\text {safe }}$ & Safe speed & $L_{\text {cell }} / \mathrm{s}$ \\
\hline$g_{\text {safety }}$ & Safety parameter to avoid accidents & $\mathrm{s}$ \\
\hline$g_{\mathrm{MV}-\mathrm{MV}}$ & Safe headway of a MV following another MV & s \\
\hline$g_{\mathrm{MV}-\mathrm{CAV}}$ & Safe headway of a MV following a CAV & $\mathrm{s}$ \\
\hline$b_{\max }$ & Maximum deceleration rate & $L_{\text {cell }} / \mathrm{s}^{2}$ \\
\hline$a_{\max }$ & Maximum acceleration rate & $L_{\text {cell }} / \mathrm{s}^{2}$ \\
\hline$d$ & Real space gap & $L_{\text {cell }}$ \\
\hline$x$ & Location at the current time step & $L_{\text {cell }}$ \\
\hline$x_{l}$ & Location of the preceding vehicle at the current time step & $L_{\text {cell }}$ \\
\hline$L_{\mathrm{veh}}$ & Length of a vehicle & $L_{\text {cell }}$ \\
\hline$v_{\text {anti }}$ & The expected velocity of the preceding vehicle & $L_{\text {cell }} / \mathrm{s}$ \\
\hline$p$ & Probability of stochastic deceleration & - \\
\hline$p_{a}, p_{b}, p_{c}$ & Probability values of stochastic deceleration & - \\
\hline$p_{\text {defense }}$ & Randomization probability & - \\
\hline$b_{\text {defense }}$ & Deceleration under defensive states & $L_{\text {cell }} / \mathrm{s}^{2}$ \\
\hline$b_{\text {rand }}$ & Randomization deceleration & $L_{\text {cell }} / \mathrm{s}^{2}$ \\
\hline$T$ & Effective safe time gap for $\mathrm{MV}$ & s \\
\hline$\alpha$ & The steepness of the logistic function $p_{\text {defense }}$ & $L_{\text {cell }} / \mathrm{s}$ \\
\hline$v_{c}$ & The midpoint of the logistic function $p_{\text {defense }}$ & $L_{\text {cell }} / \mathrm{s}$ \\
\hline$x i$ & Location at subsequent time step & $L_{\text {cell }}$ \\
\hline$v_{\mathrm{det}}^{\mathrm{CAV}}$ & Deterministic speed of CAV at subsequent time step & $L_{\text {cell }} / \mathrm{s}$ \\
\hline $\bar{v}$ & Average speed of the preceding CAVs & $L_{\text {cell }} / \mathrm{s}$ \\
\hline$K_{1}$ & Coefficient of ACC model & $\mathrm{s}^{-2}$ \\
\hline$K_{2}$ & Coefficient of ACC model & $s^{-1}$ \\
\hline$T_{\text {desired }}^{2}$ & Desired time headway between ACC-vehicle and the preceding vehicle & s \\
\hline$\Delta t$ & Delay caused by communication and detection, $\Delta t<1$ & s \\
\hline$g_{\mathrm{CAV}-\mathrm{MV}}$ & Safe headway of a CAV following a MV & s \\
\hline$g_{\mathrm{CAV}-\mathrm{CAV}}$ & Safe headway of a CAV following another CAV & s \\
\hline$d_{\text {other }}$ & The space gap between the lane-changing vehicle and vehicle immediate ahead on the target lane & $L_{\text {cell }}$ \\
\hline$d_{\mathrm{other}}^{\text {back }}$ & The space gap between the lane-changing vehicle and the immediate rear vehicle on the target lane & $L_{\text {cell }}$ \\
\hline$v_{\text {other,l }}$ & Speed of the vehicle immediately ahead on the target lane & $L_{\text {cell }} / \mathrm{s}$ \\
\hline$v_{\text {other, } r}$ & Speed of the immediate rear vehicle on the target lane & $L_{\text {cell }} / \mathrm{s}$ \\
\hline$P_{\mathrm{lc}}$ & Lane-changing probability & - \\
\hline
\end{tabular}

The subscript word "other" means the left or right adjacent lane. If the left and right lanes both meet all three conditions, the MV will seek the lane that with a higher speed. Equations (14) and (15) are proposed from the incentive aspect, while equation (16) helps to keep a safe distance after lane changing.

CAV will change to the target lane as long as the incentive equations (17) and (18) and the safety equation (19) are all met:

$$
\begin{gathered}
d+v_{l}<\min \left(v+1, v_{\max }\right), \\
d_{\text {other }}+v_{\text {other }, l}>d+v_{l}, \\
d_{\text {other }}^{\text {back }} \geq v_{\text {other }, r}-v .
\end{gathered}
$$

Note that, CAVs will degenerate to AVs if there is no CAV within the connected range (CR, normally $300 \mathrm{~m}$ ). To reduce the possibility of degeneration to AV, CAVs will choose the lane with more CAVs within CR.

\section{Scenarios with Different Exclusive Lane Policies}

In order to verify the influence of exclusive lanes setup on mixed traffic, lanes are classified into three categories, CAV exclusive lane (C), MV exclusive lane (M), and general purpose lane $(\mathrm{G})$. In which, general the purpose lane can be used by both CAVs and MVs. Considering that freeways with two and three lanes in a single direction applied most, the two-lane and three-lane freeways in a single direction are taken as cases in this study. Four lane policies for two-lane freeways (Figures 1(a) 1(d)) and twelve lane policies for three-lane freeways (Figures $1(\mathrm{e}) \sim 1(\mathrm{p})$ ) were proposed to study the traffic capacity, speed, and other characteristics. 


\begin{tabular}{ll}
\hline & \\
\hline$\square$ CAV & $\square$ General purpose lane \\
$\square$ CAV exclusive lane &
\end{tabular}

(a)

\begin{tabular}{ll}
\hline & \\
\hline CAV & $\square$ General purpose lane \\
\hline$\square$ CAV exclusive lane &
\end{tabular}

(c)

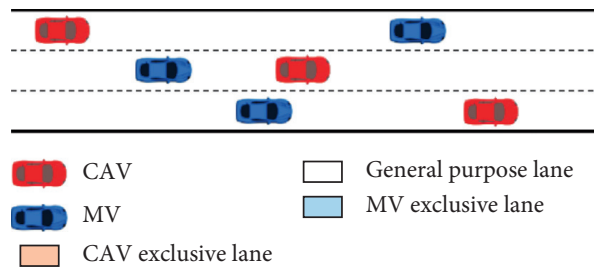

(e)

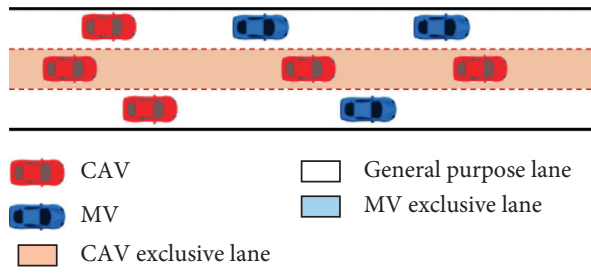

(g)

\begin{tabular}{lll}
\hline & & \\
\hline CAV & & General purpose lane \\
\hline CAV exclusive lane &
\end{tabular}

(i)

\begin{tabular}{lll}
\hline & & \\
\hline & & \\
\hline
\end{tabular}

(k)

\begin{tabular}{ll}
\hline & \\
\hline &
\end{tabular}

(m)

\begin{tabular}{ll}
\hline & \\
\hline$\square$ CAV & $\square$ General purpose lane \\
\hline MV & $\square$ MV exclusive lane \\
$\square$ CAV exclusive lane &
\end{tabular}

(b)

\begin{tabular}{ll}
\hline & \\
\hline CAV & $\square$ \\
\hline MV & $\square$ General purpose lane \\
$\square$ CAV exclusive lane &
\end{tabular}

(d)

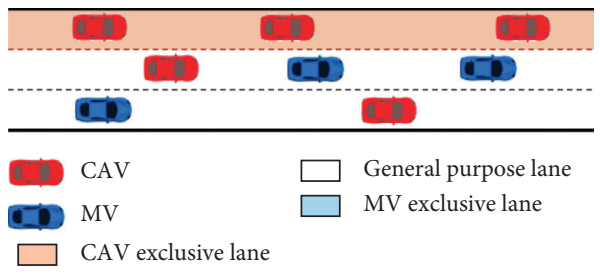

(f)

\begin{tabular}{ll}
\hline & \\
\hline &
\end{tabular}

(h)

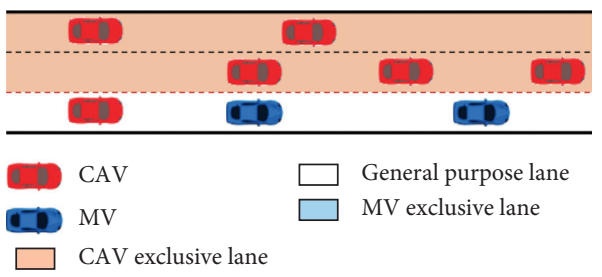

(j)

\begin{tabular}{ll}
\hline & \\
\hline &
\end{tabular}

(l)

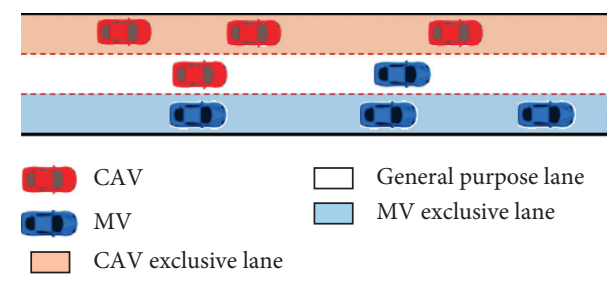

(n)

Figure 1: Continued. 


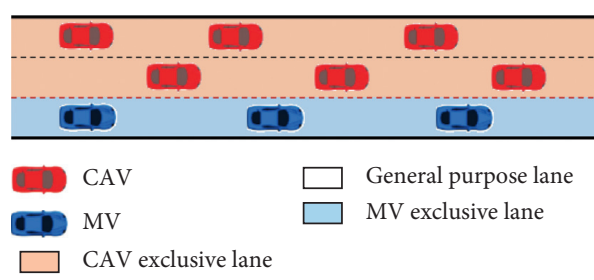

(o)

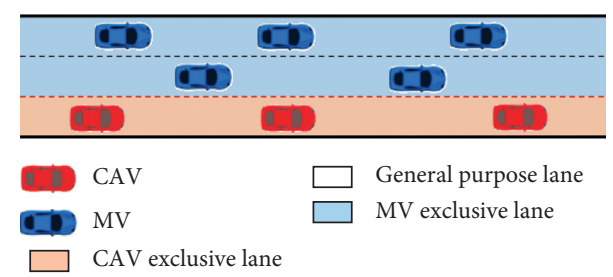

(p)

FIGUre 1: Twelve scenarios with different lane policies. (a) GG. (b) GC. (c) GM. (d) CM. (e) GGG. (f) CGG. (g) GCG. (h) MGG. (i) GMG. (j) CCG. (k) CGC. (l) MMG. (m) MGM. (n) CGM. (o) CCM. (p) MMC.

For two-lane freeways, those without exclusive lanes are denoted as GG (Figure 1(a)). In this situation, all lanes can be used by both CAVs and MVs, meaning that vehicles can change lanes freely. There is a mixed traffic of CAVs and MVs on GG.

Two-lane freeways with one exclusive lane include GC and GM. GC (Figure 1(b)) denotes that there is a CAV exclusive lane beside the general purpose (GP) lane. On this type of freeways, MVs must travel along the GP lane, while CAVs are not limited. GM (Figure 1(c)) denotes that there is a MV exclusive lane beside the GP lane. The freedom of lane changes for CAVs and MVs is opposite to that of GC. CAVs must travel along the GP lane, while MVs can change lanes freely.

Two-lane freeways with two exclusive lanes are denoted as CM (Figure $1(\mathrm{~d})$ ). In this situation, CAVs and MVs both travel along their exclusive lanes. There is no interference between CAVs and MVs.

For three-lane freeways, those without exclusive lanes are denoted as GGG (Figure 1(e)). In this situation, all lanes can be used by both CAVs and MVs, meaning that there is a mixed traffic of CAVs and MVs on these freeways. All vehicles can change lanes freely on GGG.

Three-lane freeways with one exclusive lane include CGG, GCG, MGG, and GMG. CGG (Figure 1(f)) denotes that there is a CAV exclusive lane on the left, while the remaining two lanes are GP lanes. It is worthy to note that CGG is the same with GGC. Therefore, the lane policy of GGC is not considered for simplifying the simulation process. Similar to the above, MGG (Figure 1(g)) denotes that there is a MV exclusive lane on the left, GCG (Figure $1(\mathrm{~h})$ ) denotes that there is a CAV exclusive lane between two GP lanes, and GMG (Figure 1(i)) denotes that there is a MV exclusive lane between two GP lanes. When MVs are running on the GCG, lane change is limited and MVs can only travel along one lane. The same applies to CAVs running on the GMG.

Three-lane freeways with two exclusive lanes include five policies as CCG, CGC, MMG, MGM, and CGM, respectively. CCG (Figure 1(j)) denotes that there are two CAV exclusive lanes beside a GP lane. CGC (Figure 1(k)) denotes that there is a GP lane between two CAV exclusive lanes. MVs on CCG and CGC must travel along the GP lane, and CAVs can change lanes among all lanes. MMG (Figure 1(l)) denotes that there are two MV exclusive lanes beside a GP lane. MGM (Figure $1(\mathrm{~m})$ ) denotes that there is a GP lane between two MV exclusive lanes. CAVs on MMG and GMG must travel along the GP lane and MVs can change lanes among all lanes. In addition, CGM (Figure 1(n)) denotes that there are CAV exclusive lane, GP lane, and MV exclusive lane from left to right. At this time, CAVs can change lanes between CAV exclusive lane and GP lane and MVs can change lanes between MV exclusive lane and GP lane.

Three-lane freeways with three exclusive lanes include two forms denoted as CCM and MMC. CCM (Figure 1(o)) denotes that there are two CAV exclusive lanes beside the MV exclusive lane. MMC (Figure 1(p)) denotes that there are two MV exclusive lanes beside the CAV exclusive lane. At this time, CAVs and MVs must travel along their own exclusive lanes. The lanes policies of CCC and MMM are excluded because they are not applicable to mixed traffic of CAVs and MVs.

\section{Analysis of Simulation Results}

In this section, a $6 \mathrm{~km}$ three-lane freeway segment and a $6 \mathrm{~km}$ two-lane freeway segment with periodic boundary condition were simulated. During the simulation, the cell length and vehicle length were set as $0.5 \mathrm{~m}$ and $7.5 \mathrm{~m}$, respectively, i.e., $L_{\text {cell }}=0.5 \mathrm{~m}$ and $L_{\text {veh }}=15 L_{\text {cell }}$. At each time step, a cell was occupied by a vehicle or empty. Time step was set as $1 \mathrm{~s}$. Each simulation run 5600 time steps, with the first 2000 steps being removed. The freeway was populated up with mixed CAVs and MVs at the beginning. Since the initial location and speed of vehicles were random, some differences in results were expected with each simulation run. To address this issue, each simulation was run 5 times. The reported simulation result, then, was the average of 5 runs. All the lanes policies mentioned in Section 4 were simulated and compared.

The parameter values of MVs and CAVs are listed in Tables 2 and 3, respectively. For MV, most of the parameters follow the ones in [1], which was calibrated with data from I-80 freeway, CA, USA. The safe headway between two MVs, $g_{\mathrm{MV}-\mathrm{MV}}$, was set as $1.8 \mathrm{~s}$, in accordance with the Highway Capacity Manual (HCM) estimates. The safe headway when a MV runs after a CAV is a bit larger, i.e. $2.4 \mathrm{~s}$, representing a more defensive driving behavior. With more confidence in the CAV in the future, $g_{\mathrm{MV}-\mathrm{CAV}}$ will decrease gradually and be smaller than $g_{\mathrm{MV}-\mathrm{MV}}$ at last.

Due to the lack of field data of CAVs, the parameters of ACC in equation (10) are from [1]. The $300 \mathrm{~m}$ of the 
TABle 2: Parameters for MVs.

\begin{tabular}{lcccccccccccc}
\hline Parameters & $T$ & $a$ & $b_{\max }$ & $b_{\text {defense }}$ & $p_{\mathrm{a}}$ & $p_{\mathrm{b}}$ & Pc & $g_{\mathrm{MV}-\mathrm{MV}}$ & $g_{\mathrm{MV}-\mathrm{CAV}}$ & $v_{c}$ & $\alpha$ & $P_{\mathrm{lc}}$ \\
\hline Values & 1.8 & 1 & 3 & 1 & 0.85 & 0.52 & 0.1 & 1.8 & 2.4 & 30 & 10 & 0.2 \\
\hline
\end{tabular}

TABle 3: Parameters for CAVs.

\begin{tabular}{lcccccccccc}
\hline Parameters & $\mathrm{CR}$ & $P_{\mathrm{lc}}$ & $T_{\mathrm{ACC}}$ & $\Delta t$ & $a_{\max }$ & $K_{1}$ & $K_{2}$ & $g_{\mathrm{CAV}-\mathrm{MV}}$ & $g_{\mathrm{CAV}-\mathrm{CAV}}$ \\
\hline Values & 300 & 0.2 & 0.5 & 0.1 & 3 & 0.14 & 0.90 & 0.9 & 0.0 \\
\hline
\end{tabular}

connected range was widely adopted in the previous studies [37]. Communication delay was set as $0.1 \mathrm{~s}$, which is defensive to avoid a possible accident. The safe headway between two CAVs was $0 \mathrm{~s}$, while that between a CAV and a MV was $0.9 \mathrm{~s}$.

5.1. Flow-Density Relationship with Different Lane Policies. The relationship between traffic flow and its density is illustrated (Figure 2). Figures 2(a) 2(d) illustrate the two-lane freeways, while the others show the three-lane freeways. Figures 2(a) and 2(e) show the freeways with no exclusive lane, while Figures 2(b) 2(d), 2(f) 2(n), 2(o), and 2(p) are freeways with one exclusive lane, two exclusive lanes, and three exclusive lanes, respectively.

When the lanes are all GP lanes, as shown in Figures 2(a) and 2(e), the flow increases to a peak and then decreases gradually. If the density remains constant, the flow will become larger with the increase of $P_{\mathrm{CAV}}$. During the free flow phase, the increase of $P_{\mathrm{CAV}}$ can enlarge the density range. As a result, the flow peak, known as capacity, will be greater and appears under a larger density, if the value of $P_{\text {CAV }}$ increases. When the vehicles are all connected and autonomous, the two-lane freeway capacity reaches nearly $4100 \mathrm{veh} / \mathrm{ln} / \mathrm{h}$ with a density of $47 \mathrm{veh} / \mathrm{ln} / \mathrm{km}$. While for three-lane freeways with $100 \% \mathrm{CAV}$, the capacity is over $4100 \mathrm{veh} / \mathrm{ln} / \mathrm{h}$ when the density is $43 \mathrm{veh} / \mathrm{ln} / \mathrm{km}$. Note that, instead of growth, the density with the largest flow unexpected drops when $P_{\mathrm{CAV}}$ changes from 0.9 to 1 .

When one lane is exclusive, it can be for CAVs or for MVs. For two-lane freeway segments, a CAV exclusive lane is of great use to increase the road capacity, when compared with an MV exclusive lane. In Figure 2(b), the flow peaks increase at least $10 \%$ when $P_{\mathrm{CAV}}$ is no smaller than 0.3 . As few CAVs exist when $P_{\mathrm{CAV}}$ is equal to 0.1 , a CAV exclusive lane is a kind of road space waste and will result in a reduction in flow peak. When the exclusive lane is for MVs, as shown in Figure 2(c), no benefit can be observed, in terms of flow peak increase. However, the situation for three-lane freeways is a bit complicated. It is obvious in Figures $2(\mathrm{f})$ and $2(\mathrm{~g})$, the flow peaks increase generally, especially when $P_{\mathrm{CAV}}$ is no smaller than 0.5 . When $P_{\mathrm{CAV}}$ is small, the introduction of CAV lane will reduce the road capacity. Note that when the density is from $40 \mathrm{veh} / \mathrm{ln} / \mathrm{km}$ to $80 \mathrm{veh} / \mathrm{ln} / \mathrm{km}$; the flow remains stable if $P_{\mathrm{CAV}}$ is equal to 0.3 or 0.5 . This is quite different from GGG. When the exclusive lane is for MVs, the flow is diminished due to the low opportunity to gain a higher speed for CAVs. It is worthy to mention that, when
$P_{\mathrm{CAV}}$ is small (Figures 2(f) 2(g)) and when $P_{\mathrm{CAV}}$ is big (Figures 2(h) 2(i)), no dot can be observed if the density is larger than $90 \mathrm{veh} / \mathrm{ln} / \mathrm{km}$. It appears because the existence of the exclusive lane for a kind of specific vehicles means a capacity reduction of the other kind of vehicles.

When two exclusive lanes are considered, the impact of exclusive lanes on traffic flow is more significant. For two-lane freeways, two exclusive lanes indicate a CAV exclusive lane, an MV exclusive lane, and no general purpose lane. As lane changing is strictly forbidden under the circumstances, a larger flow peak can be achieved only if the number of CAVs and MVs are close. As shown in Figure 2(d), the maximum flow peak appears when $P_{\mathrm{CAV}}$ is 0.5 . For three-lane freeways, the flow peaks further increase if two CAV exclusive lanes exist and $P_{\mathrm{CAV}}$ is no smaller than 0.5 , as shown in Figures 2(j) 2(k). While in Figures 2(l) 2(m), flow peak drop to under $2000 \mathrm{veh} / \mathrm{ln} / \mathrm{h}$, even if only $10 \%$ of vehicles are connected and autonomous. In addition, the largest density when CGC (and CCG) with small $P_{\mathrm{CAV}}$, or MGM (and MMG) with large $P_{\mathrm{CAV}}$, is reduced to $60 \mathrm{veh} / \mathrm{ln} / \mathrm{km}$. In Figure 2(n), two exclusive lanes are allocated for CAVs and MVs separately. The flow is generally between the CGC (or CCG) policy and MGM (or MMG) policy if the density and $P_{\mathrm{CAV}}$ remain the same.

In Figures 2(o) 2(p), all three lanes are exclusive. In Figure 2(o), the number of CAV exclusive lanes is more, which makes the flow peaks pretty high if $P_{\mathrm{CAV}}$ remains big. The largest flow peak here is very close to those in Figures 2(j) 2(k). Under these lane policies, freeways are all with two CAV exclusive lanes. In Figure 2(p), although the number of $\mathrm{MV}$ exclusive lanes is the same as those in Figures 2(l) 2(m), the flow peaks increase. Remarkably, the largest flow in Figure 2(o) appears when $P_{\mathrm{CAV}}$ is 0.7 rather than 0.9. Again, the largest flow in Figure 2(p) appears when $P_{\mathrm{CAV}}$ is 0.3 rather than 0.1 . Both are quite different.

In Figure 2, the increase of CAV exclusive lanes can improve the flow peaks, especially when $P_{\mathrm{CAV}}$ is more than 0.5. MV exclusive lanes and, however, seem helpless for road capacity improvement.

To further investigate the road capacity, Tables 4 and 5 illustrate the flow peaks for different lane policies and $P_{\mathrm{CAV}}$. In these tables, the cells in one column in italics, bold, and underline indicate the capacity ranks $1^{\text {st }}, 2^{\text {nd }}$, and $3^{\text {rd }}$ under a certain $P_{\mathrm{CAV}}$, respectively.

In Table 4, GG outperforms the others when $P_{\mathrm{CAV}}$ is no bigger than 0.2 . The capacity gap between GG and GC is tiny when $P_{\mathrm{CAV}}$ is 0.1 and 0.2 . However, the introduction of an MV exclusive lane can greatly reduce the capacity. When 

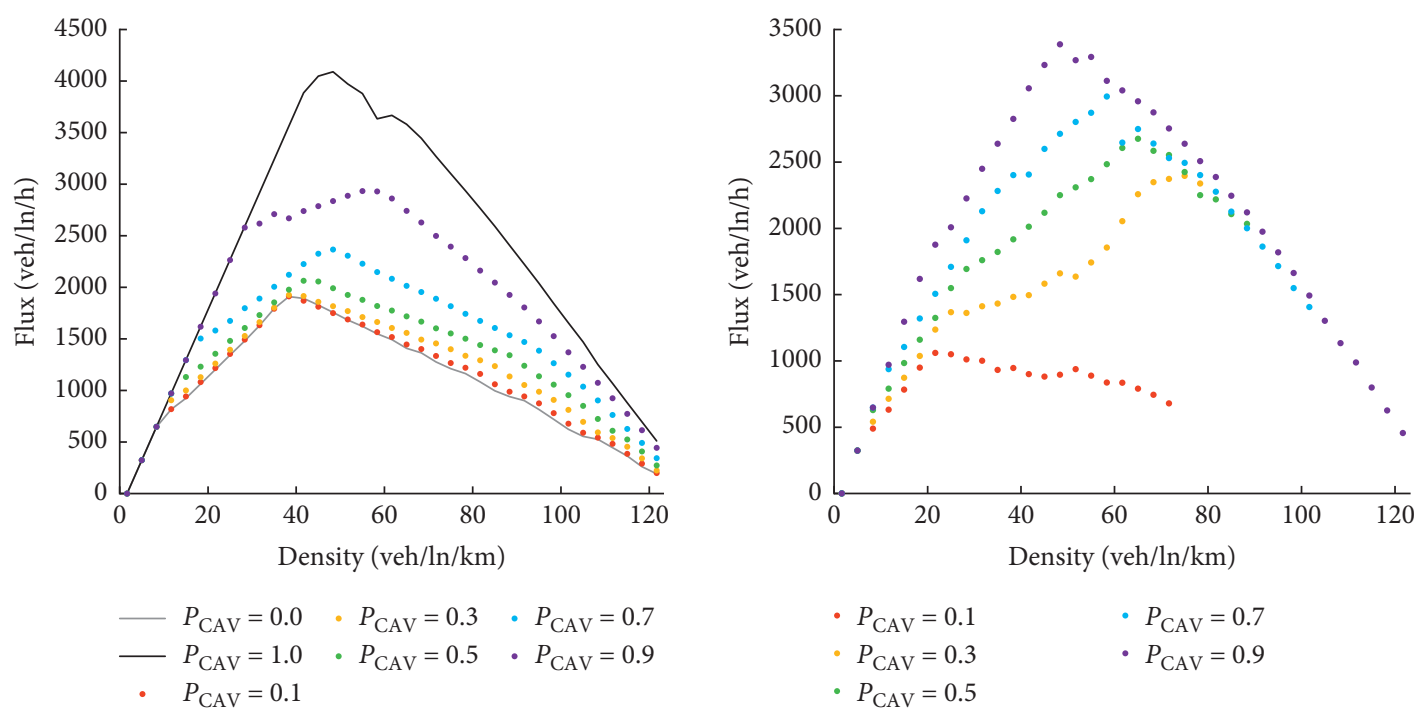

(a)

(b)
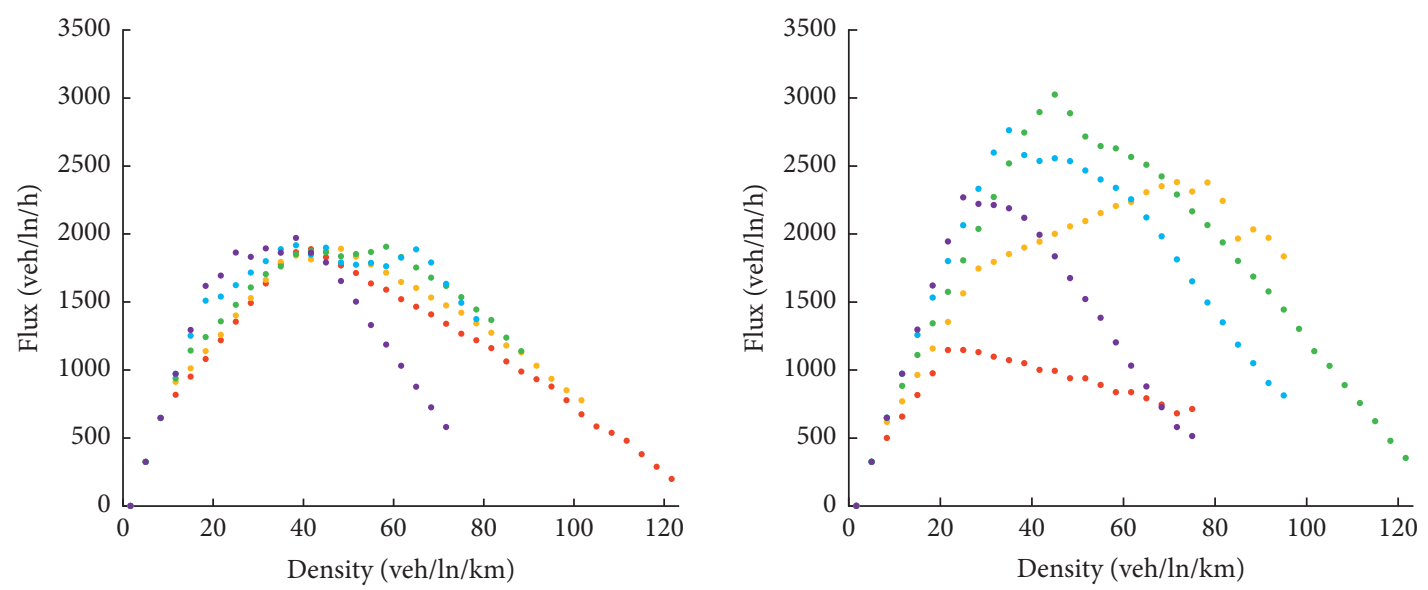

$$
\begin{array}{ll}
-P_{\mathrm{CAV}}=0.1 & \cdot P_{\mathrm{CAV}}=0.7 \\
-P_{\mathrm{CAV}}=0.3 & \cdot P_{\mathrm{CAV}}=0.9 \\
\text { - } P_{\mathrm{CAV}}=0.5 &
\end{array}
$$

(c)

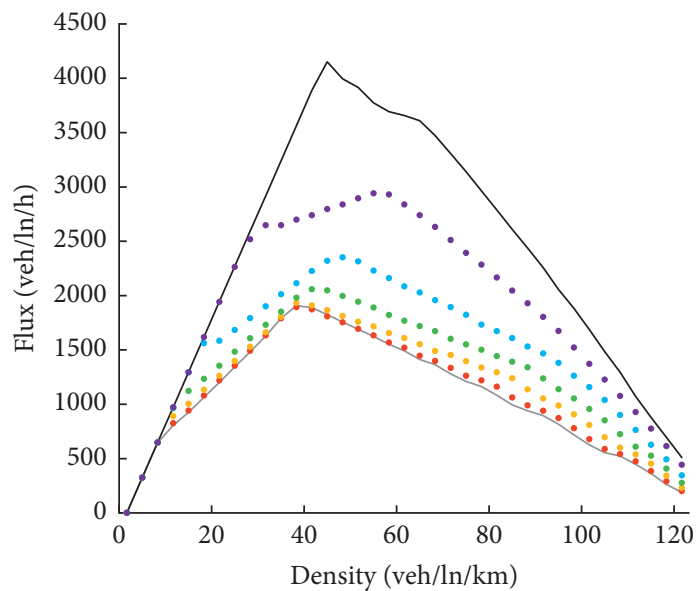

$$
\begin{array}{lll}
-P_{\mathrm{CAV}}=0.0 & \cdot P_{\mathrm{CAV}}=0.3 & \cdot P_{\mathrm{CAV}}=0.7 \\
-P_{\mathrm{CAV}}=1.0 & \cdot P_{\mathrm{CAV}}=0.5 & \cdot P_{\mathrm{CAV}}=0.9 \\
-P_{\mathrm{CAV}}=0.1 & &
\end{array}
$$

(e)

$$
\begin{array}{ll}
-P_{\mathrm{CAV}}=0.1 & \cdot P_{\mathrm{CAV}}=0.7 \\
-P_{\mathrm{CAV}}=0.3 & \cdot P_{\mathrm{CAV}}=0.9 \\
\text { - } P_{\mathrm{CAV}}=0.5 &
\end{array}
$$

(d)

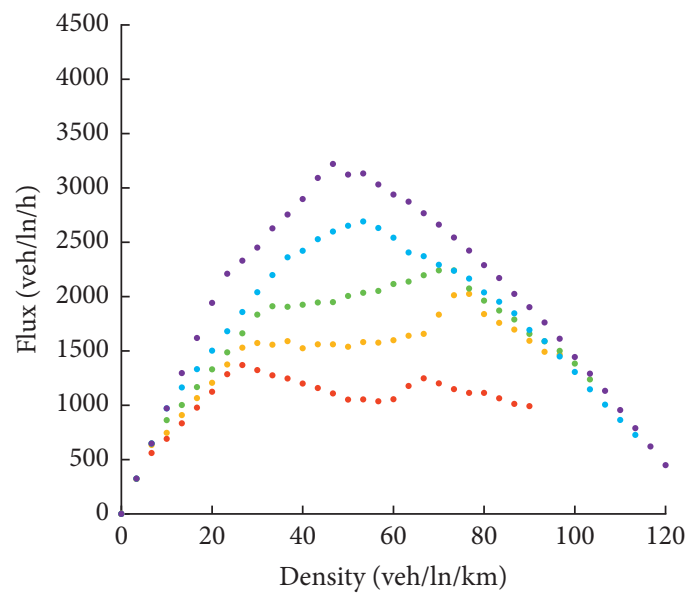

$$
\begin{array}{ll}
-P_{\mathrm{CAV}}=0.0 & \cdot P_{\mathrm{CAV}}=0.7 \\
\cdot P_{\mathrm{CAV}}=0.3 & \cdot P_{\mathrm{CAV}}=0.9 \\
\cdot P_{\mathrm{CAV}}=0.5 &
\end{array}
$$

(f)

FIgURE 2: Continued. 

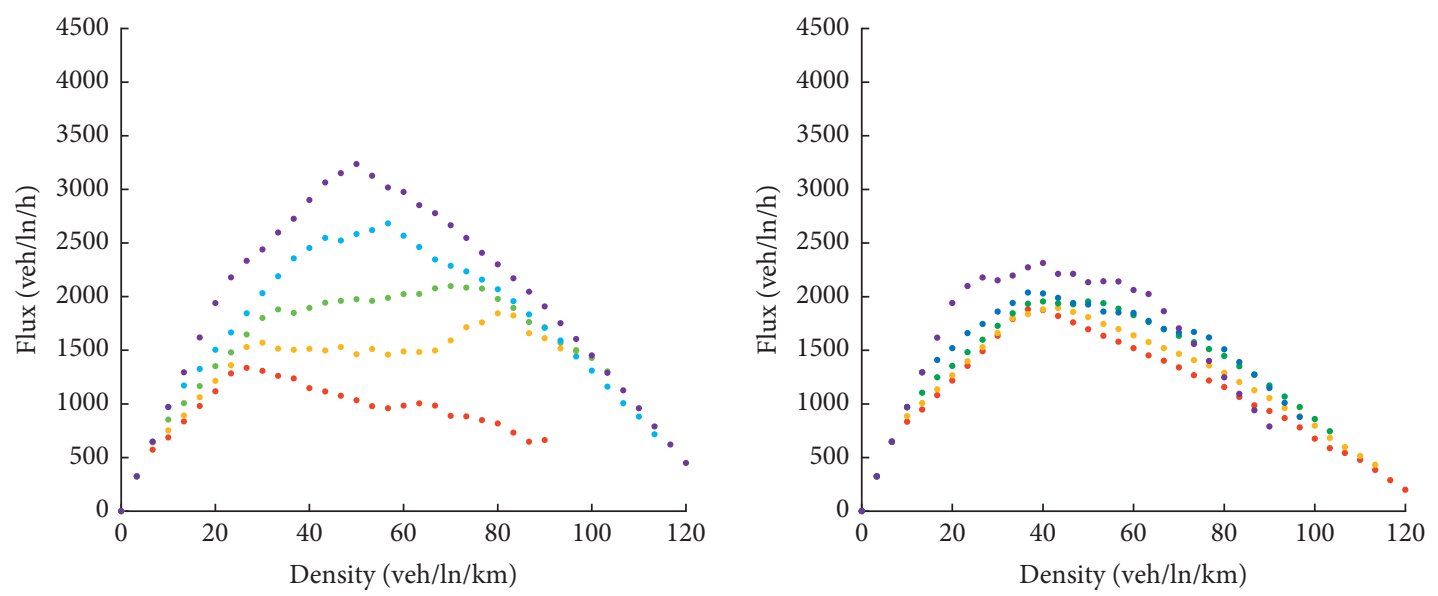

$$
\begin{array}{ll}
-P_{\mathrm{CAV}}=0.1 & \cdot P_{\mathrm{CAV}}=0.7 \\
\text { - } P_{\mathrm{CAV}}=0.3 & \cdot P_{\mathrm{CAV}}=0.9 \\
\text { - } P_{\mathrm{CAV}}=0.5 &
\end{array}
$$

(g)

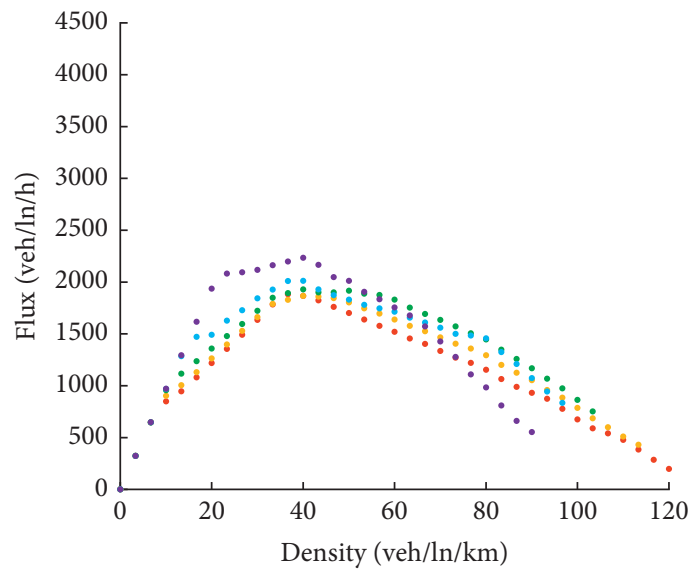

$$
\begin{aligned}
& \text { - } P_{\mathrm{CAV}}=0.1 \\
& -P_{\mathrm{CAV}}=0.3 \\
& \text { - } P_{\mathrm{CAV}}=0.5
\end{aligned}
$$

$$
\begin{aligned}
& \cdot P_{\mathrm{CAV}}=0.7 \\
& \cdot P_{\mathrm{CAV}}=0.9
\end{aligned}
$$

(i)

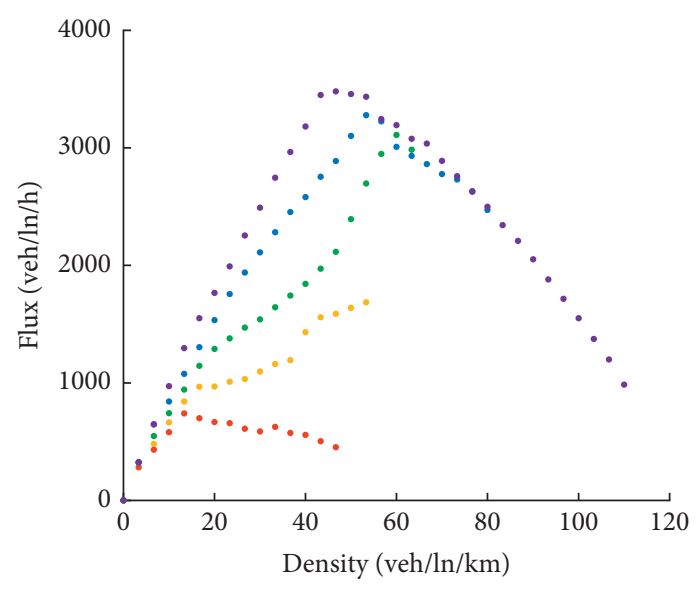

$$
\begin{array}{ll}
\text { - } P_{\mathrm{CAV}}=0.1 & \cdot P_{\mathrm{CAV}}=0.7 \\
\text { - } P_{\mathrm{CAV}}=0.3 & \cdot P_{\mathrm{CAV}}=0.9 \\
\text { - } P_{\mathrm{CAV}}=0.5 &
\end{array}
$$

(h)

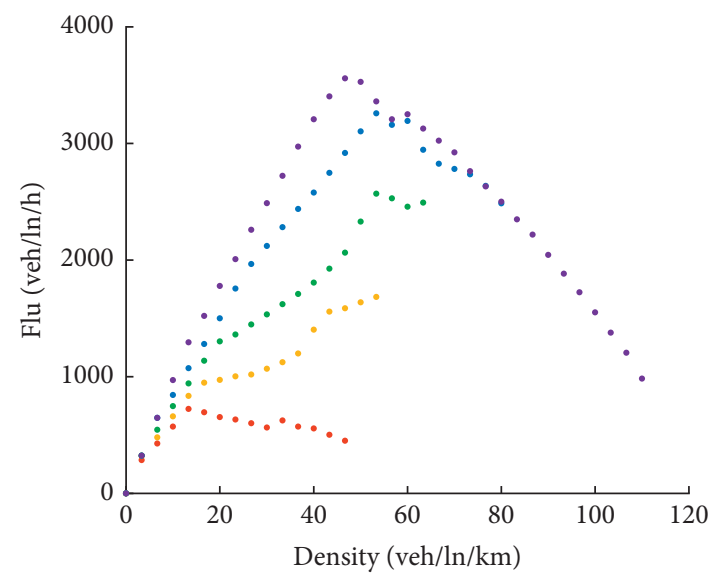

$$
\begin{array}{ll}
-P_{\mathrm{CAV}}=0.1 & \cdot P_{\mathrm{CAV}}=0.7 \\
\cdot P_{\mathrm{CAV}}=0.3 & \cdot P_{\mathrm{CAV}}=0.9 \\
\cdot P_{\mathrm{CAV}}=0.5 &
\end{array}
$$

(j)

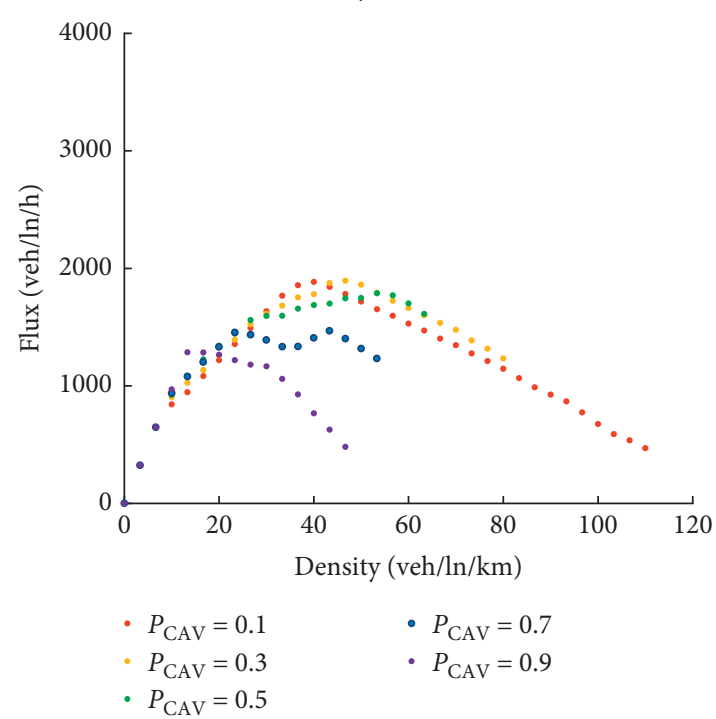

(k)

(1)

Figure 2: Continued. 


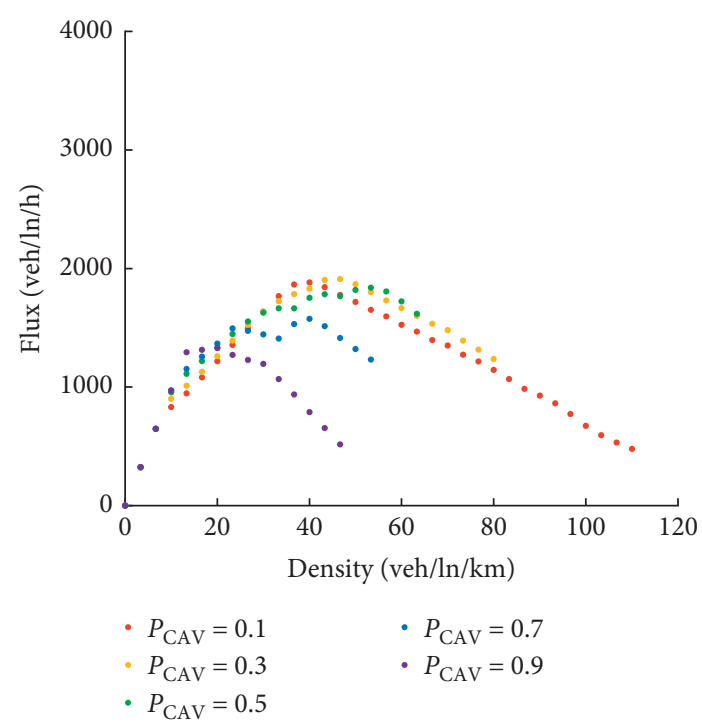

(m)

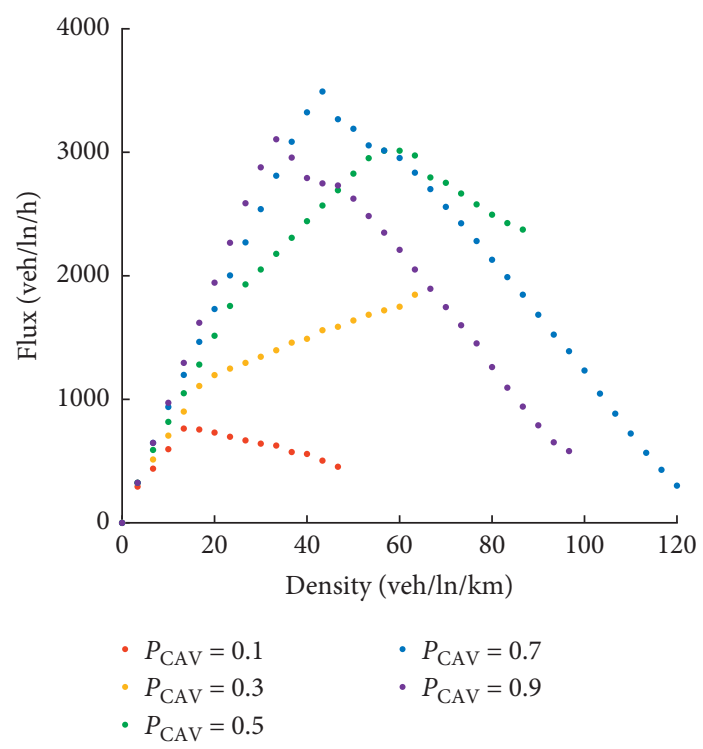

(o)

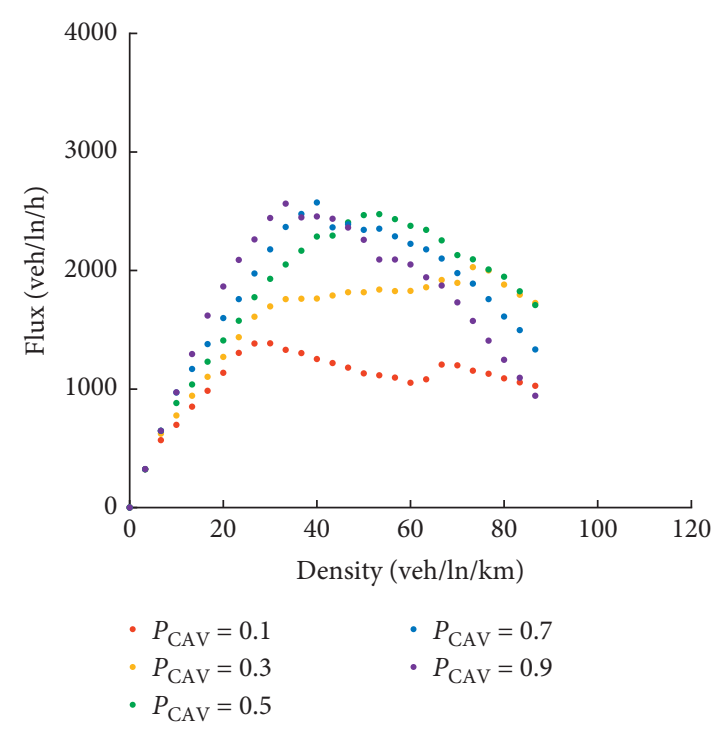

(n)

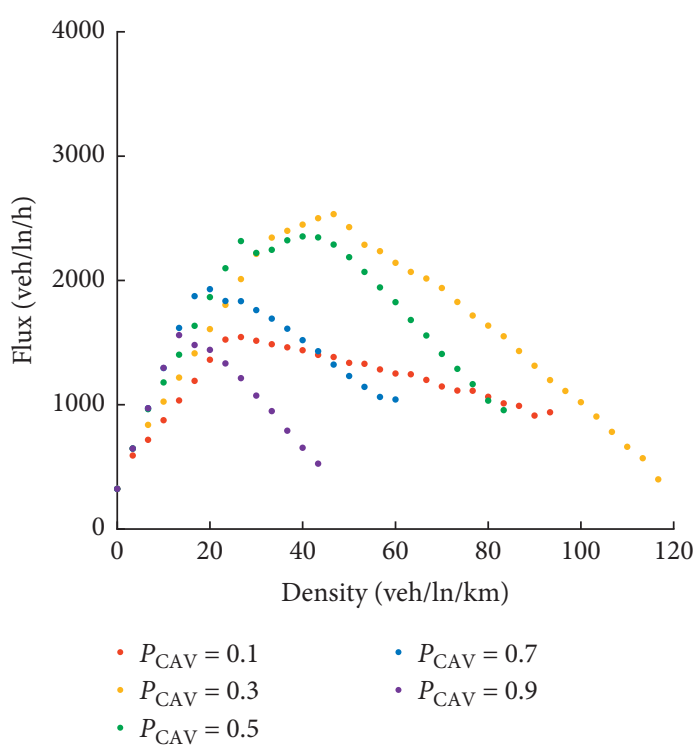

(p)

FIgure 2: Flow-Density relationship diagram with different lane policies. (a) GG. (b) GC. (c) GM. (d) CM. (e) GGG. (f) CGG. (g) GCG. (h) MGG. (i) GMG. (j) CCG. (k) CGC. (l) MMG. (m) MGM. (n) CGM. (o) CCM. (p) MMC.

$P_{\mathrm{CAV}}$ is over 0.3 , CM policy becomes the best in terms of capacity. When $P_{\mathrm{CAV}}$ is larger than $0.7, \mathrm{GC}$ outperforms the others.

For three-lane freeways, as shown in Table 5, it is obvious GGG outperforms the others only if $P_{\mathrm{CAV}}$ is 0.1 . The capacity gaps among GGG, MMG, and GGM are all very small when $P_{\text {CAV }}$ is 0.1 . When one lane is exclusive, GCG seems better in terms of capacity, especially when $P_{\mathrm{CAV}}$ equals 0.2 and 03 . Although CGG ranks $3^{\text {rd }}$ when $P_{\mathrm{CAV}}$ is set as 0.9 , the capacity is only $0.5 \%$ larger than that of GCG. For policies with two exclusive lanes, CGC and CCG both perform well when $P_{\text {CAV }}$ is no less than 0.4. CGM is pretty good as well when $P_{\mathrm{CAV}}$ is 0.2 and 0.3 . For policies with three exclusive lanes, CCM is better when $P_{\mathrm{CAV}}$ is no less than 0.4 while MMC outperforms the others when $P_{\mathrm{CAV}}$ is 0.2 and 0.3 . Overall, in terms of capacity, when $P_{\mathrm{CAV}}$ is no less than $0.4, \mathrm{MMC}$, GCG, and CGM are recommended, while if $P_{\mathrm{CAV}}$ is over 0.5 , CCM, CGC, and CCG are better.

5.2. Average Speed Performance Analysis. In addition to flux, the average speed is another measure to evaluate the traffic performance of different lane policies. In this part, the average speed under various traffic density was compared and the speed difference was calculated, to better understand the advantage of each policy (Figure 3).

For two-lane freeways with one exclusive lane, it is hard to tell whether GC or GM performs better in terms of travel speed. In Figure 3(a), when $P_{\mathrm{CAV}}$ is small, GM can get a higher travel speed, while when $P_{\mathrm{CAV}}$ is over $0.5, \mathrm{GC}$ 
TABle 4: Road capacity with different $P_{\mathrm{CAV}}$ values (two-lane freeways).

\begin{tabular}{|c|c|c|c|c|c|c|c|c|c|}
\hline \multirow{2}{*}{$\begin{array}{l}\text { Lane } \\
\text { policies }\end{array}$} & \multicolumn{9}{|c|}{$P_{\mathrm{CAV}}$} \\
\hline & 0.1 & 0.2 & 0.3 & 0.4 & 0.5 & 0.6 & 0.7 & 0.8 & 0.9 \\
\hline & & & & & J & & 6 & & \\
\hline GL & & 60 & 2381 & 2551 & 2676 & $\underline{2775}$ & 2995 & 3082 & 3388 \\
\hline GM & 1887 & 1874 & 1890 & 1919 & 1906 & 1892 & 1917 & 1934 & 1971 \\
\hline $\mathrm{CM}$ & $\overline{1147}$ & $\overline{1569}$ & 2396 & 2772 & 3025 & 2956 & $\underline{2763}$ & 2479 & 2268 \\
\hline
\end{tabular}

TABle 5: Road capacity with different $P_{\text {CAV }}$ values (three-lane freeways).

\begin{tabular}{lccccccccc}
\hline $\begin{array}{l}\text { Lane } \\
\text { policies }\end{array}$ & 0.1 & 0.2 & 0.3 & 0.4 & 0.5 & 0.6 & 0.7 & 0.8 & 0.9 \\
\hline GGG & 1894 & 1904 & 1932 & 1989 & 2058 & 2180 & 2352 & 2588 & 2940 \\
CGG & 1337 & 1469 & 1844 & 1948 & 2098 & 2463 & 2682 & 2906 & $\underline{3236}$ \\
GCG & 1369 & $\mathbf{1 9 5 0}$ & $\underline{2024}$ & 2079 & 2240 & 2468 & 2691 & 2954 & 3220 \\
GGM & $\underline{1883}$ & 1879 & 1866 & 1886 & 1930 & 1919 & 2012 & 2140 & 2235 \\
GMG & 1882 & 1895 & 1894 & 1907 & 1956 & 1966 & 2039 & 2174 & 2314 \\
CCG & 725 & 1037 & 1685 & 2324 & 2570 & $\mathbf{3 1 1 7}$ & $\underline{3259}$ & 3381 & 3558 \\
CGC & 739 & 1037 & 1685 & $\underline{2451}$ & $\underline{3110}$ & $\underline{3106}$ & $\mathbf{3 2 7 8}$ & $\mathbf{3 3 2 9}$ & $\mathbf{3 4 8 1}$ \\
CGM & 1385 & $\underline{1907}$ & $\mathbf{2 0 2 8}$ & 2253 & 2475 & $\mathbf{2 6 1 0}$ & 2573 & 2616 & 2563 \\
MGM & 1882 & 1882 & 1910 & 1904 & 1837 & 1800 & 1575 & 1463 & 1329 \\
MMG & $\mathbf{1 8 8 5}$ & 1891 & 1894 & 1915 & 1788 & 1628 & 1469 & 1369 & 1286 \\
CCM & 764 & 1037 & 1847 & 2814 & $\mathbf{3 0 1 4}$ & 3239 & 3493 & $\underline{3237}$ & 3105 \\
MMC & 1545 & 2022 & 2534 & $\mathbf{2 6 3 4}$ & 2354 & 2028 & 1930 & 1803 & 1560 \\
\hline
\end{tabular}

becomes better. The largest travel speed gap between GC and GM is over $20 \mathrm{~km} / \mathrm{h}$ when $P_{\mathrm{CAV}}$ is 0.9 and traffic density is near $50 \mathrm{veh} / \mathrm{ln} / \mathrm{km}$. Besides, we also compared GC with GG, as shown in Figure 2(b). The speed difference shows a similar trend, i.e., GC outperforms GG only when freeways are with enough CAVs.

For two-lane freeways with two exclusive lanes, the travel speed on CM is compared with that on GG. CM policy performs better when $P_{\mathrm{CAV}}$ is equal to $0.3,0.5$, and 0.7 and GG performs better under other penetrations.

For three-lane freeways with one exclusive lane, performance in travel speed of GCG is better than that of CGG overall, especially when $P_{\mathrm{CAV}}$ is less than 0.5 (Figure 3(d)). Traffic speed of GCG and CGG is similar under traffic density from $80 \mathrm{veh} / \mathrm{ln} / \mathrm{km}$ to $120 \mathrm{veh} / \mathrm{ln} / \mathrm{km}$. Furthermore, we also compared GCG with GGG (Figure 3(e)). The speed difference shows that GCG performs better when $P_{\mathrm{CAV}}$ is more than 0.5 . When traffic density is more than $80 \mathrm{veh} / \mathrm{ln} / \mathrm{km}$, GCG performs better under all $P_{\mathrm{CAV}}$, but the difference is narrowing gradually with the increase of traffic density. Similarly, performance in travel speed of GMG is better than that of MGG overall, especially when $P_{\mathrm{CAV}}$ is more than 0.5 (Figure 3(f)). Travel speed of GMG and MGG is similar under various traffic densities when $P_{\mathrm{CAV}}$ is less than 0.5. However, the comparison between GGG and GMG shows that GMG has no advantage except when $P_{\mathrm{CAV}}$ is small (Figure $3(\mathrm{~g})$ ).

For three-lane freeways with two exclusive lanes, performance in travel speed of CGC is better than that of CCG overall and speed difference between CGC and CCG is small (Figure 3(h)). CGC was compared with GGG (Figure 3(i)), which shows that CGC performs better when $P_{\mathrm{CAV}}$ is more than 0.5 and the advantage of CGC is more obvious during traffic density is about $50 \mathrm{veh} / \mathrm{ln} / \mathrm{km}$. On the other hand, the average speed on MGM is better than that on MMG when $P_{\text {CAV }}$ is more than 0.5 (Figure $3(\mathrm{j})$ ). The average speed on MGM and MMG is nearly equal when traffic density is more than $60 \mathrm{veh} / \mathrm{ln} / \mathrm{km}$. Then, we compared average speed on GGG and that on MGM. The result (Figure 3(k)) shows that GGG performs better under $P_{\mathrm{CAV}}$ which is more than 0.5 and there is little difference between GGG and MGM in other cases.

For three-lane freeways with all exclusive lanes, we only compared travel speed on GGG with that on CCM and found that CCM performs better when $P_{\mathrm{CAV}}$ is equal to 0.5 and 0.7 and GGG performs better under other penetrations (Figure 3(l)).

In conclusion, the best lane policies in terms of the average travel speed are GC, GM, and CM for two-lane freeways and GCG, CGC, and CCM for three-lane freeways, respectively.

To further investigate the best lane policies with the largest average speed, a colored diagram is illustrated (Figure 4), in which, colors denote the lane policies and the numbers denote the speed difference between the best policy and its following policy. The characteristics are concluded as follows: (1) GG and GGG perform best when $P_{\mathrm{CAV}}$ is small. The CAV exclusive lane set can be considered with the increase of $P_{\mathrm{CAV}}$. In addition, there is little speed difference between GG/GGG and other policies when traffic density is low and $P_{\mathrm{CAV}}$ is big as the upper right corner of Figure 4. Under this situation, GG/GGG is also accepted for lanes set. (2) GM performs best only when $P_{\text {CAV }}$ is small and density ranges from $15 \mathrm{veh} / \mathrm{ln} / \mathrm{km}$ to around $100 \mathrm{veh} / \mathrm{ln} / \mathrm{km}$. The advantage range is limited. The advantage range of GC is larger when compared with that of GM policy. GC performs best when $P_{\mathrm{CAV}}$ is no less than 0.4 . (3) The advantage of GCG is obvious when traffic density is more than $65 \mathrm{veh} / \mathrm{ln} / \mathrm{km}$. The speed difference between GCG and its following policy is narrowing with the increase of $P_{\mathrm{CAV}}$. When traffic density and $P_{\mathrm{CAV}}$ increase at the same time, it is recommendable to adopt GCG for better average travel speed. (4) The advantage range of CGC is limited. CGC becomes the best form when $P_{\mathrm{CAV}}$ is over 0.4. The higher the $P_{\mathrm{CAV}}$ is, the larger range of travel density where CGC performs best will be. (5) $\mathrm{CM}$ and CCM are applicable to the scene where traffic density is not big and $P_{\mathrm{CAV}}$ is not small. In this time, CAVs and MVs travel along their exclusive lanes and have noninterference with each other.

5.3. Average Speed Comparison between CAVs and MVs. In this subsection, the average speeds of CAVs and MVs were compared. The best lane policies in terms of average speed, GC, GM, CM, GCG, CGC, and CCM were selected. GG/GGG was neglected here because the fully mixed traffic of CAVs and MVs made the average speeds very close. To describe the speed gap between CAVs and MVs, a ratio $r$ was defined as follows:

$$
r=\frac{\overline{v_{\mathrm{CAV}}}}{\overline{v_{\mathrm{MV}}}} .
$$




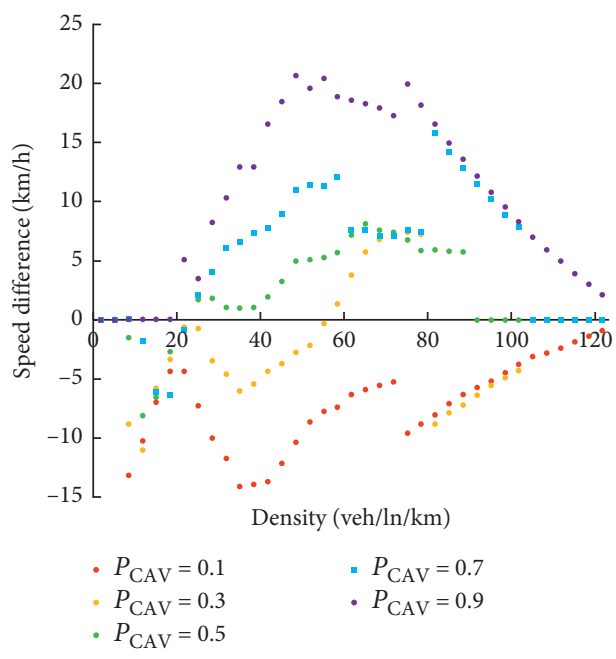

(a)

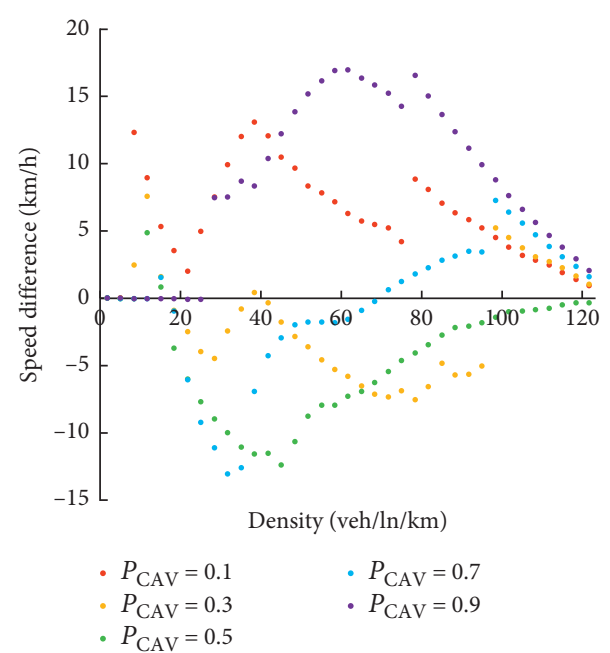

(c)

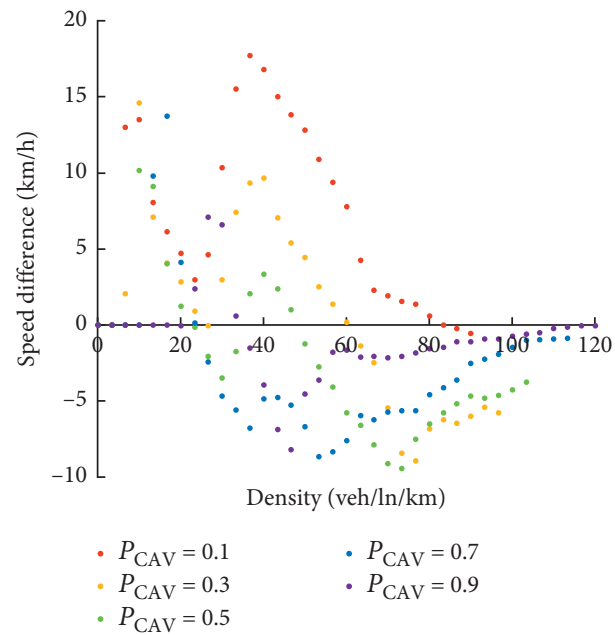

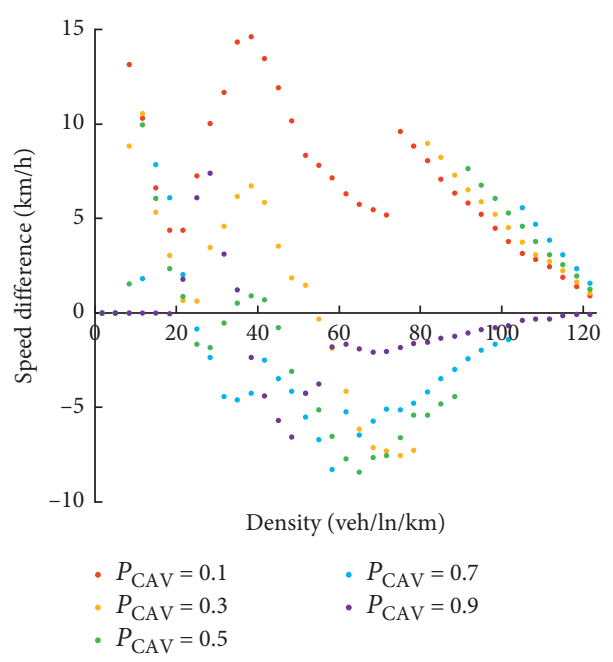

(b)

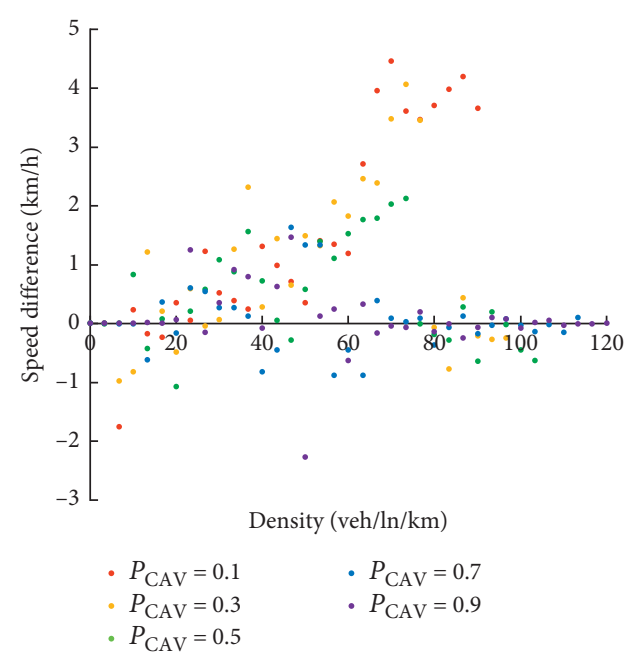

(d)

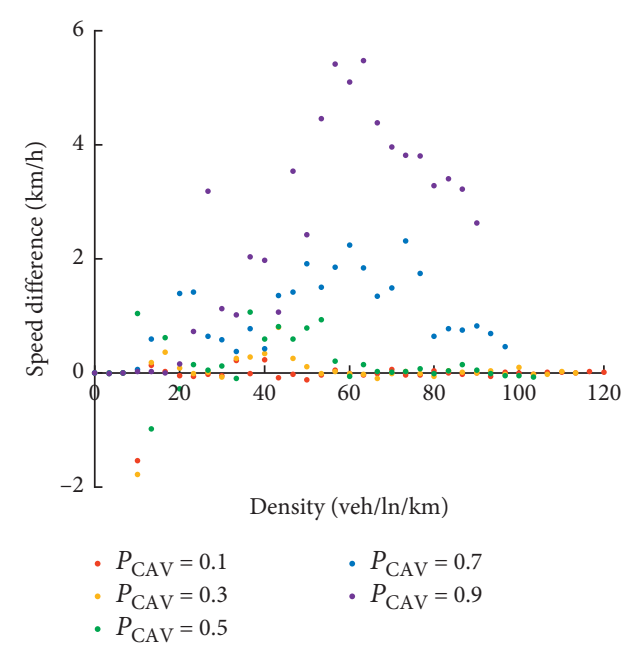

(f)

FIgURE 3: Continued. 

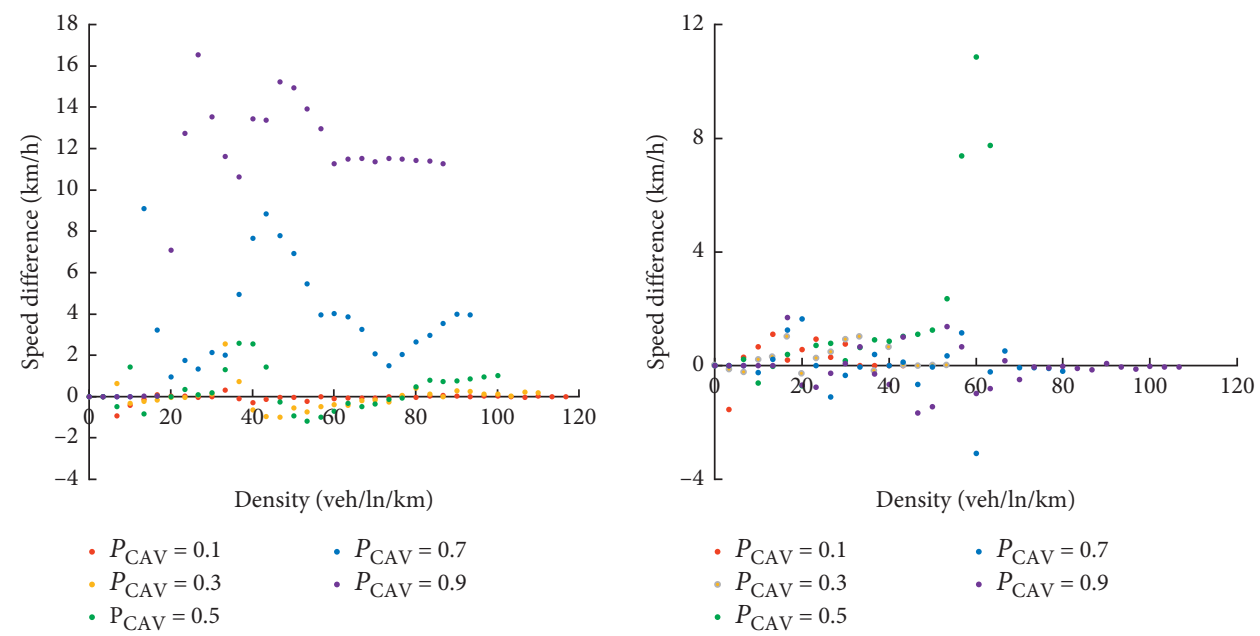

(g)

(h)
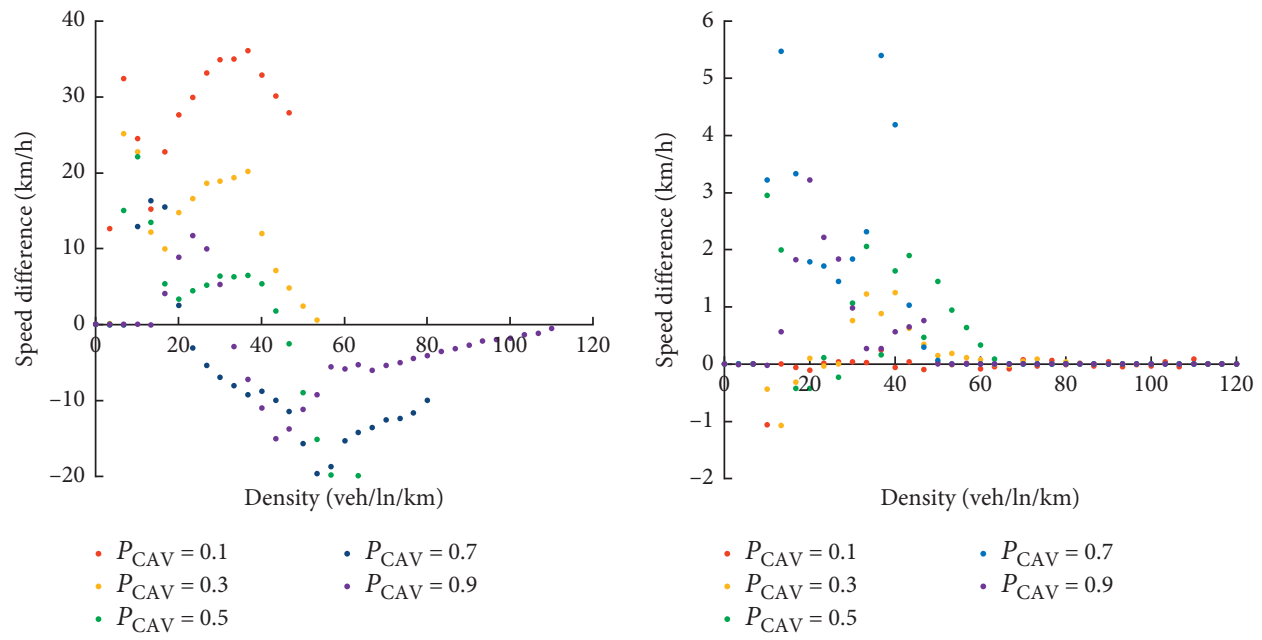

(i)

(j)

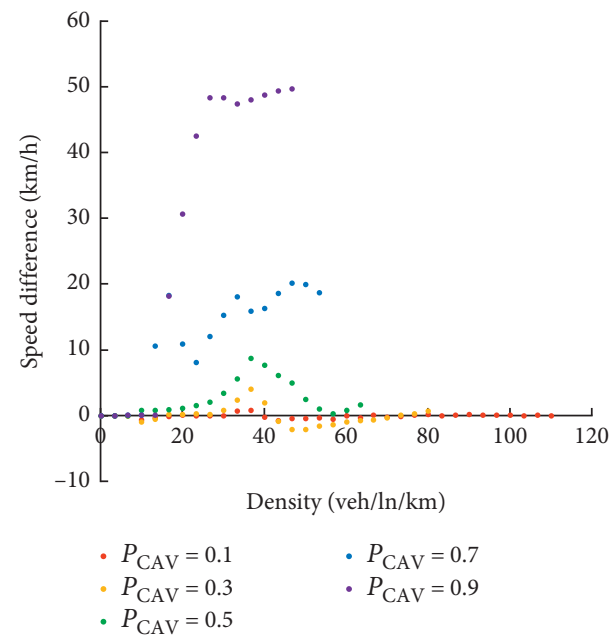

$(\mathrm{k})$

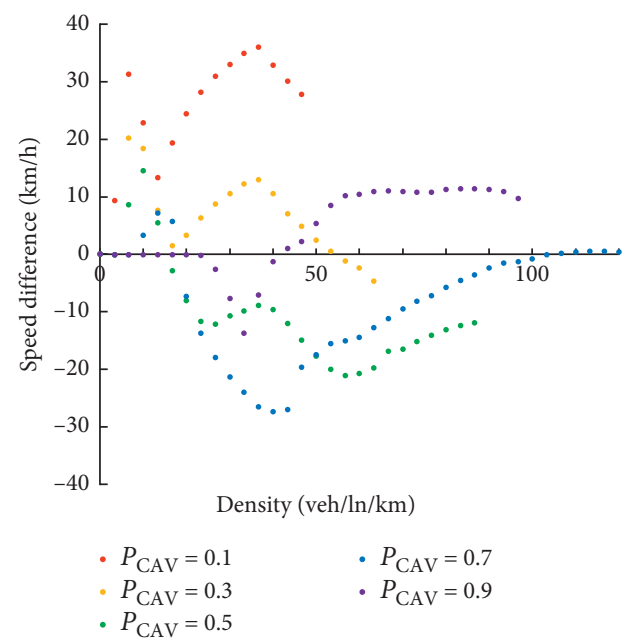

(l)

Figure 3: Speed different diagram. Speed difference between (a) GC and GM, (b) GG and GC, (c) GG and CM, (d) GCG and CGG, (e) GGG and GCG, (f) GMG and MGG, (g) GGG and GMG, (h) CGC and CCG, (i) GGG and CGC, (j) MGM and MMG, (k) GGG and MGM, and (l) GGG and CCM. 


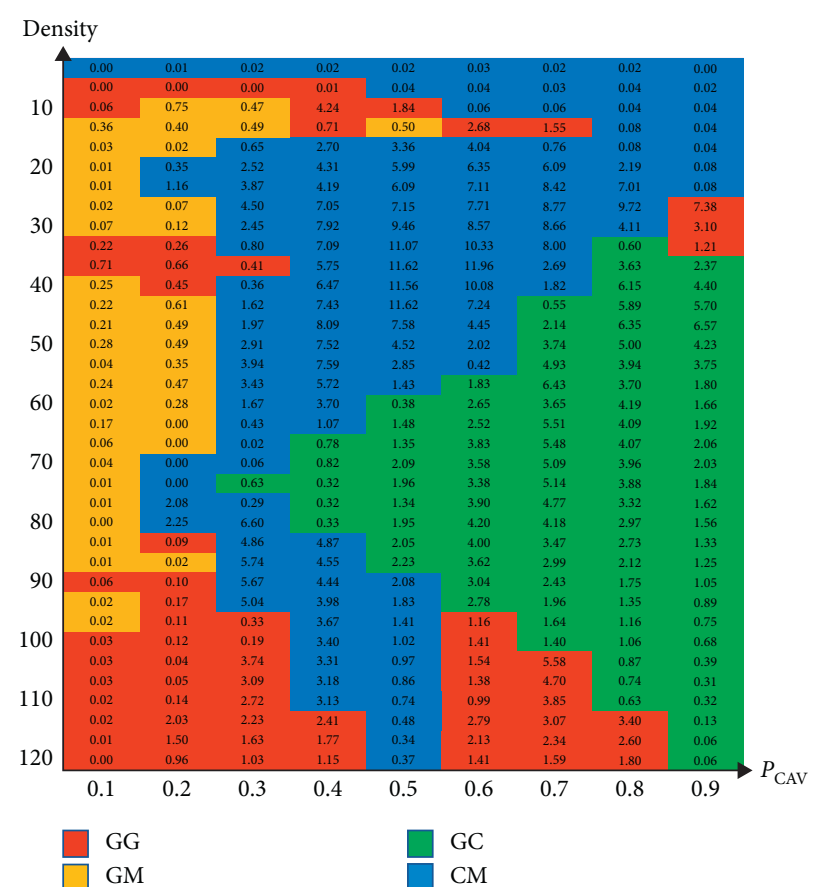

(a)

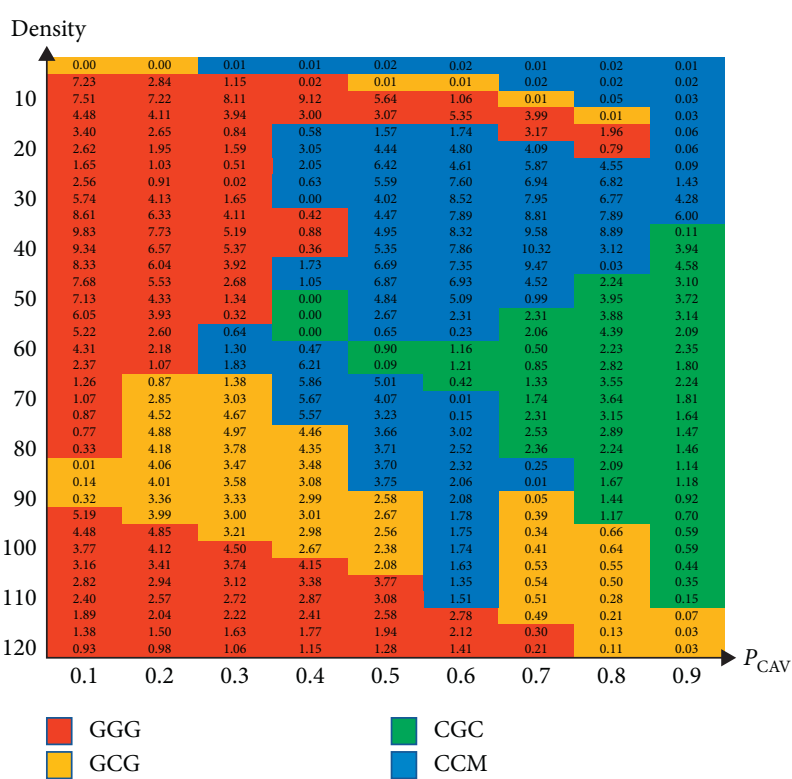

(b)

Figure 4: (a) Diagram of best lane policies in terms of average speed (two-lane freeways). (b) Diagram of best lane policies in terms of average speed (three-lane freeways).

The change of ratio $r$ in density and $P_{\mathrm{CAV}}$ is shown (Figure 5). Domains I, II, and III indicate that the ranges of $r$ are $(0,2],(2,5],(5,10]$, and $(10, \infty)$, respectively. The grey domain IV means the road is overloaded in this situation. From the upper right to the lower left, the values of $r$ increase, indicating a larger density and smaller $P_{\mathrm{CAV}}$ can lead to a greater speed gap between CAVs and MVs.

The areas of domains II and III in Figures 5(a)-5(f) are quite similar, but the domain I changes a lot. For GC, the domain I is about 1.9 times the area of domains II and III, while for CM the domain I is about 1.5 times the area of domains II and III. For GCG, the domain I is about 1.9 times the area of domains II and III. For CGC and CCM, the domain $\mathrm{I}$ is about 0.8 times area and 1.5 times the area of domains II and III, respectively. Remarkably, for GM policy in Figure 5(b), only two domains can be observed, i.e., domains I and IV. It is hard to say whether a larger $r$ is good or not. However, from the aspect of CAV popularization, a larger $r$ will hopefully result in a tendency to travel with a higher speed CAV rather than using MV. This is helpful to increase the penetration rate of CAVs.

5.4. Analysis of Degradation ofCAVs. Except for the flow and speed, the degradation rate of CAVs is another indicator. In order to illustrate the transformation of CAV degradation rate, a bar diagram is adopted, as shown in Figure 6. In this figure, traffic density was set as $40 \mathrm{veh} / \mathrm{ln} / \mathrm{km}$ considering that this value is close to traffic capacity. The proportions of each type of vehicle on GG, GC, GM, CM, GGG, GCG, CGC, and $\mathrm{CCM}$ were obtained under various $P_{\mathrm{CAV}}$.
In Figure 6, CAV represents the front vehicle is still a CAV and $\mathrm{AV}$ denotes the front vehicle is MV. No vehicle within CR means the front vehicle is too far away. The results are shown as follows: (1) exclusive lanes set can enlarge the proportion of CAVs and reduce the proportion of degradation to AVs. The more exclusive lanes are, the better the effect is. (2) lanes can be used evenly on GG/GGG and no vehicle within CR does not exist. Besides, no vehicle within CR does not exist for GM policy as well. In addition, AVs do not exist on CM/CCM because there are no MVs ahead of CAVs. (3) There are no AVs on CGC when $P_{\mathrm{CAV}}$ is low, indicating CAVs mainly travel along the exclusive lanes. However, AVs come into being with the increase of CAVs, on the ground that CAVs move onto the GP lane for better travel speed. The number of CAVs which move onto the GP lane is increasing generally, leading to an increasing proportion of AVs. But when $P_{\mathrm{CAV}}$ is over 0.6, the distance between CAVs is shortened and the proportion of AVs comes to decline. (4) CM/CCM can ensure that CAVs do not become AVs. When $P_{\mathrm{CAV}}$ is over 0.6 , the proportion of CAVs is close to 1 .

\section{Discussion}

In this section, the influence of some importation factors on mixed traffic and lane policies were investigated. The factors included safe headway and lane-changing probability. A $6 \mathrm{~km}$ three-lane freeway segment with periodic boundary condition was simulated as well. The simulation model and its parameter values used in this section were the same as in Section 5. Four lanes policies, i.e., GGG, GCG, CGC, and CCM, were simulated and evaluated. 


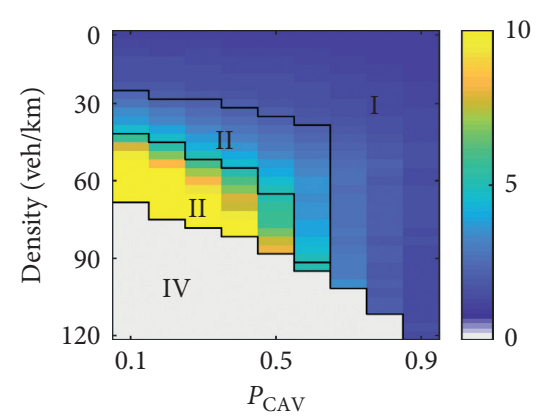

(a)

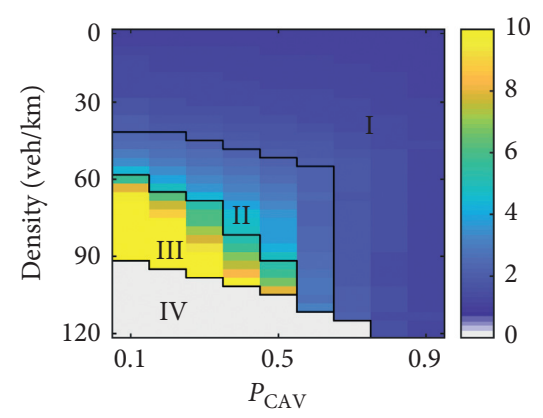

(d)

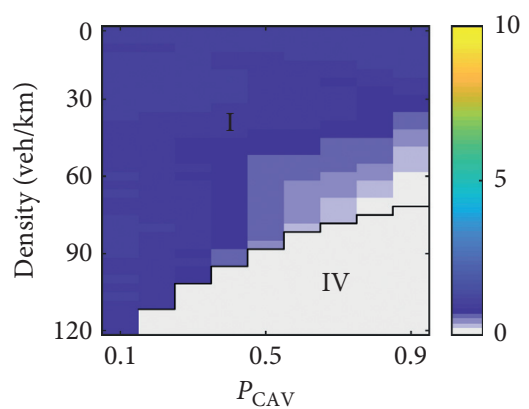

(b)

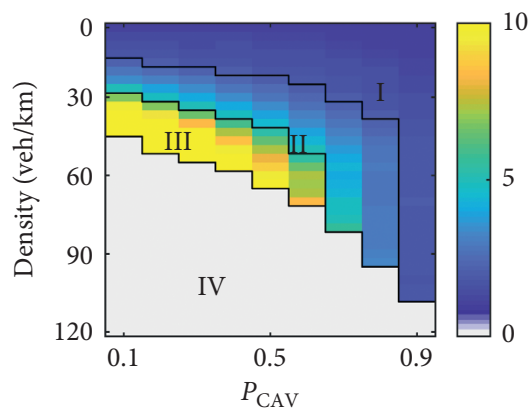

(e)

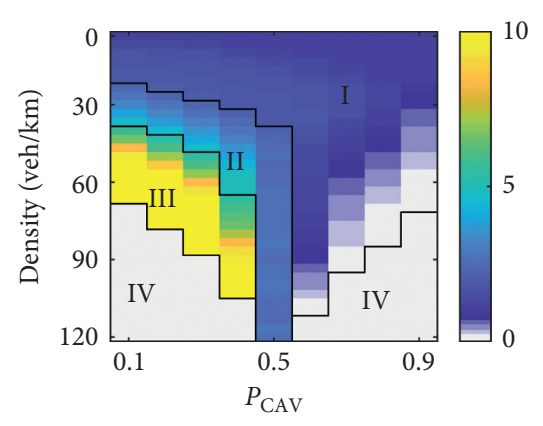

(c)

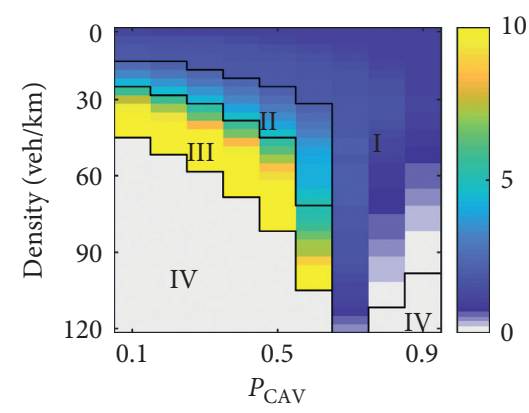

(f)

Figure 5: The diagram of ratio $r$. (a) GC. (b) GM. (c) CM. (d) GCG. (e) CGC. (f) CCM.

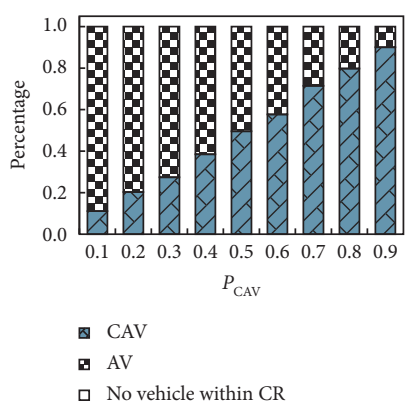

(a)

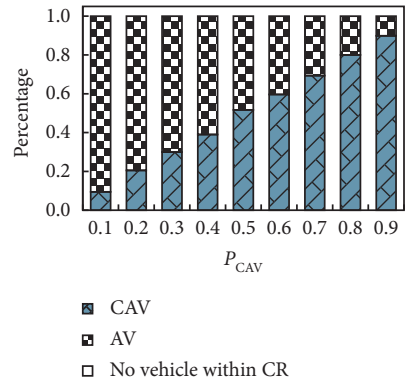

(e)

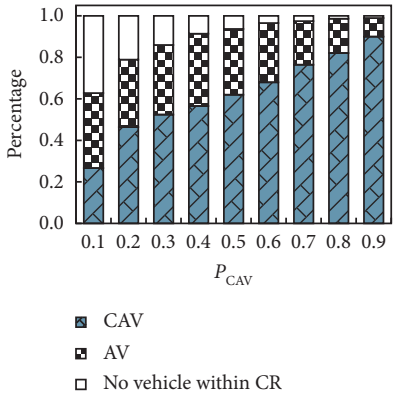

(b)

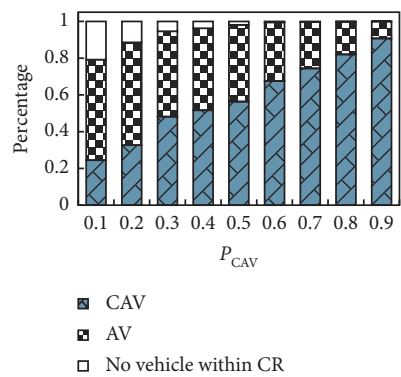

(f)

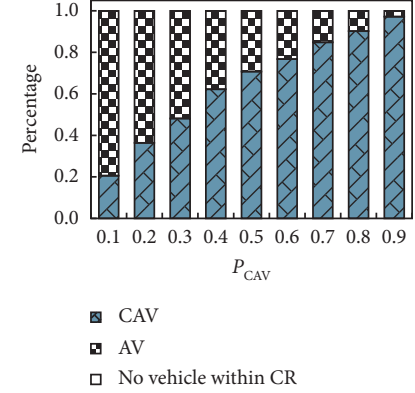

(c)

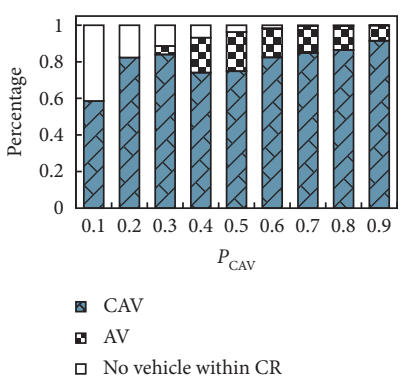

(g)

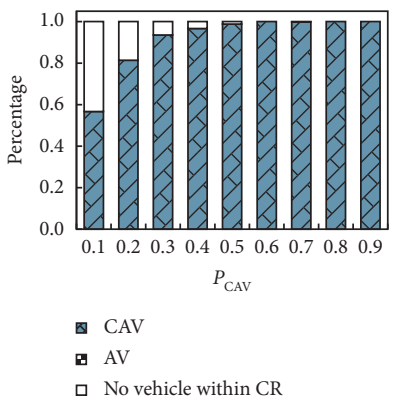

(d)

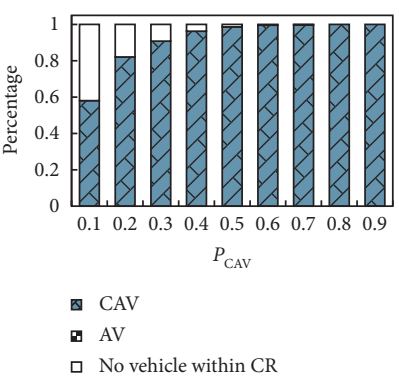

(h)

Figure 6: The percentage of each state of CAVs. (a) GG. (b) GC. (c) GM. (d) CM. (e) GGG. (f) GCG. (g). CGC. (h) CCM.

6.1. Impact of Safe Headway on Capacity. It is no surprise when only MVs exist, the increase in capacity is largely based on the improvement of individual vehicle performance, in terms of a decrease in headway. In this subsection, the safe headway will be varied, to check the impact of safe headway on capacity. Figure 7 shows the capacity under four lane policies with various $g_{\mathrm{CAV}-\mathrm{MV}}$ and $g_{\mathrm{MV}-\mathrm{CAV}}$ values.

From Figure 7, safe headway can affect road capacity. For freeways without exclusive lanes (GGG in Figure 7(a)), the decrease of $g_{\mathrm{MV} \text {-CAV }}$ can significantly increase the road capacity no matter what the value of $P_{\mathrm{CAV}}$ is. However, the 

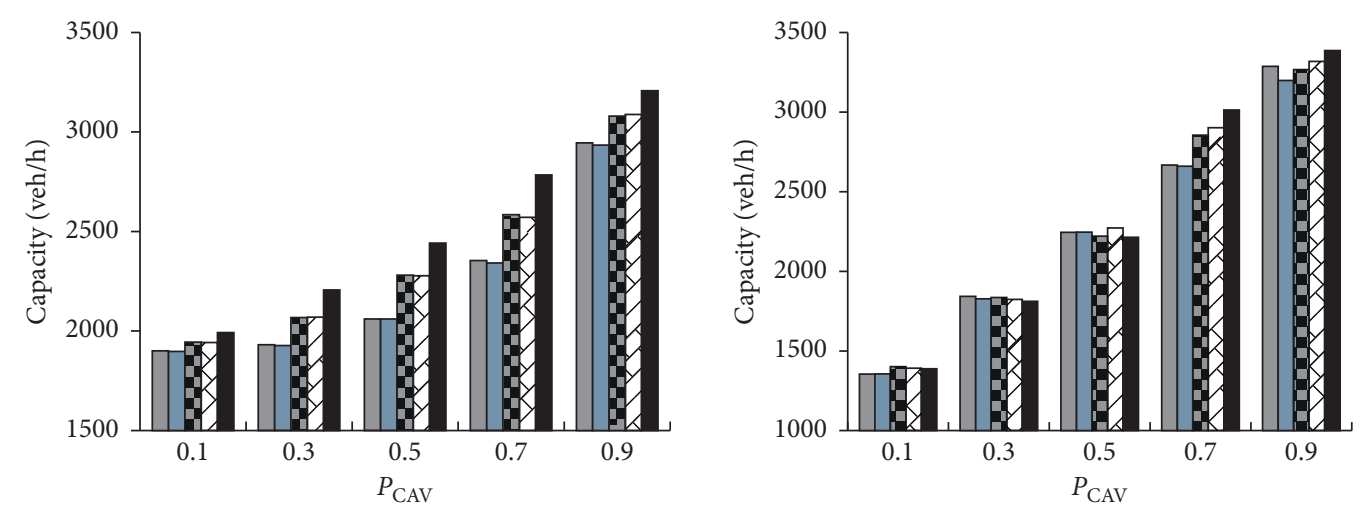

ㅁ $g_{\mathrm{MV}-\mathrm{CAV}}=2.4 \mathrm{~s}, g_{\mathrm{CAV}-\mathrm{MV}}=0.9 \mathrm{~s}$
ㅁ $g_{\mathrm{MV}-\mathrm{CAV}}=2.4 \mathrm{~s}, g_{\mathrm{CAV}-\mathrm{MV}}=0.3 \mathrm{~s}$
․ $g_{\mathrm{MV}-\mathrm{CAV}}=1.8 \mathrm{~s}, g_{\mathrm{CAV}-\mathrm{MV}}=0.9 \mathrm{~s}$
ㅁ $g_{\mathrm{MV}-\mathrm{CAV}}=1.8 \mathrm{~s}, g_{\mathrm{CAV}-\mathrm{MV}}=0.3 \mathrm{~s}$
- $g_{\mathrm{MV}-\mathrm{CAV}}=1.2 \mathrm{~s}, g_{\mathrm{CAV}-\mathrm{MV}}=0.3 \mathrm{~s}$

(a)

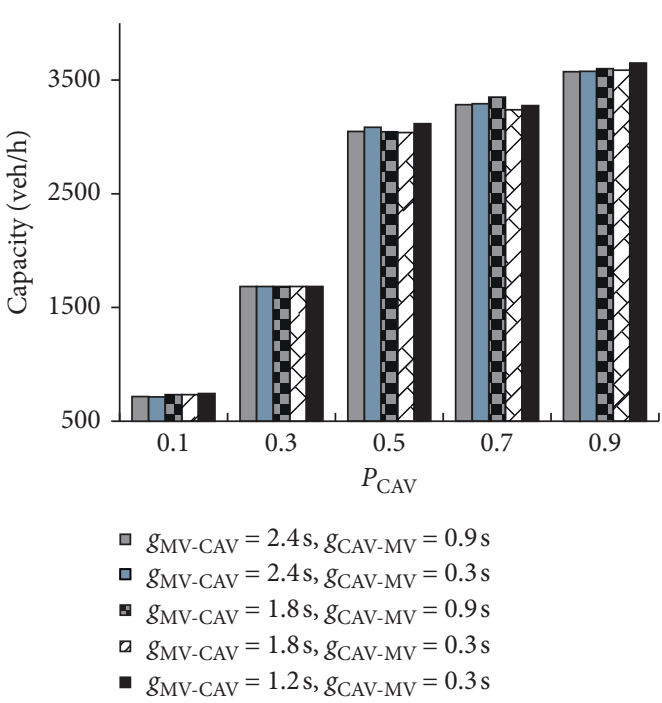

(c)

$$
\begin{aligned}
& \text { g } g_{\mathrm{MV}-\mathrm{CAV}}=2.4 \mathrm{~s}, g_{\mathrm{CAV}-\mathrm{MV}}=0.9 \mathrm{~s} \\
& \square g_{\mathrm{MV}-\mathrm{CAV}}=2.4 \mathrm{~s}, g_{\mathrm{CAV}-\mathrm{MV}}=0.3 \mathrm{~s} \\
& g_{\mathrm{MV}-\mathrm{CAV}}=1.8 \mathrm{~s}, g_{\mathrm{CAV}-\mathrm{MV}}=0.9 \mathrm{~s} \\
& g_{\mathrm{MV}-\mathrm{CAV}}=1.8 \mathrm{~s}, g_{\mathrm{CAV}-\mathrm{MV}}=0.3 \mathrm{~s} \\
& g_{\mathrm{MV}-\mathrm{CAV}}=1.2 \mathrm{~s}, g_{\mathrm{CAV}-\mathrm{MV}}=0.3 \mathrm{~s}
\end{aligned}
$$

(b)

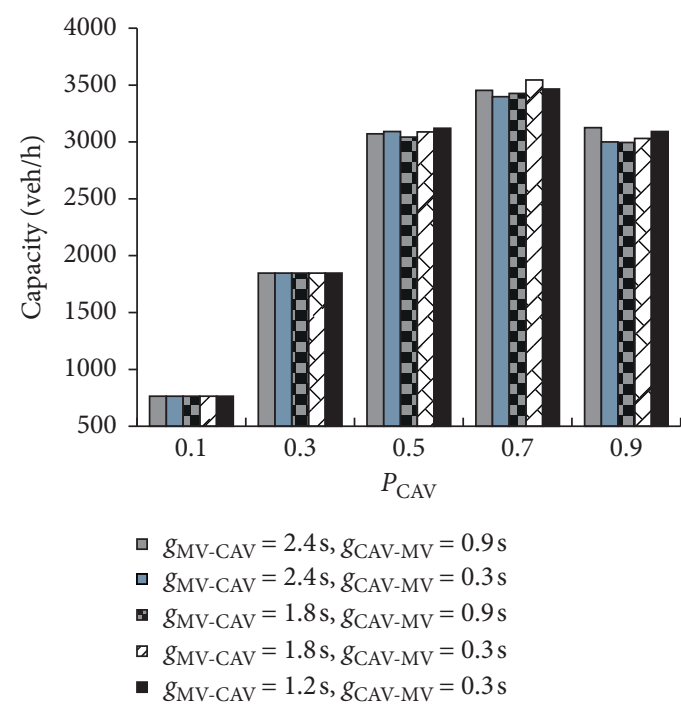

(d)

FIGURE 7: Relationship between capacity and CAV penetration rate with different $g_{\mathrm{CAV}-\mathrm{MV}}$ and $g_{\mathrm{MV}-\mathrm{CAV}}$ (a) GGG. (b) GCG. (c). CGC. (d) CCM.

impact of $g_{\text {CAV-MV }}$ on capacity is very slight and different from that of $g_{\mathrm{MV}-\mathrm{CAV}}$. The road capacity may decrease with a smaller $g_{\mathrm{CAV}-\mathrm{MV}}$. In Figures $7(\mathrm{~b})-7(\mathrm{~d})$ with CAV exclusive lanes, the impact of safe headway $g_{\mathrm{CAV}-\mathrm{MV}}$ and $g_{\mathrm{MV}-\mathrm{CAV}}$ on capacity is slighter, especially when $P_{\mathrm{CAV}}$ is smaller than 0.5 . When $P_{\mathrm{CAV}}$ is over 0.7 , the decrease of $g_{\mathrm{MV}-\mathrm{CAV}}$ can increase the road capacity on the whole.

Generally, with no CAV exclusive lane, the decrease of $g_{\text {MV-CAV }}$ can significantly increase the road capacity, while the decrease of $g_{\text {CAV-MV }}$ can have a negative impact on capacity. When freeways are with CAV exclusive lanes, the impact of safe headway changes on capacity is not obvious, especially when CAV penetration rate is small.

6.2. Impact of Lane-Changing Probability of CAVs on Average Speed and Capacity. The lane change behaviors in multilane freeways may help to obtain a higher and more stable flow speed. Here, we get the average speeds when the lanechanging probability of CAVs is $0.2,0.6$, and 1.0 . The speeds when $P_{\mathrm{lc}}=0.2$ are set as benchmark speeds, and speeds when $P_{\mathrm{lc}}=0.6$ and 1.0 are for comparison. Figure 8 illustrates the speed changes over the benchmark. In the figure, speed changes over 0 indicate an increase, while the negative values mean speed decrease compared with benchmark speeds.

Form Figure 8, it is obvious that the increase of lanechanging probability of CAVs can increase the average freeway speed, especially when the freeway consists of both exclusive lanes and general purpose lanes, as shown in Figures 8 (c)-8(f). The larger $P_{\mathrm{lc}}$ for CAVs, the higher average freeway speed will be. Besides, larger speed increases are more likely to be observed when the density is smaller than $70 \mathrm{veh} / \mathrm{km} / \mathrm{ln}$. However, for freeways with no exclusive lane or with no general purpose lane, as shown in 


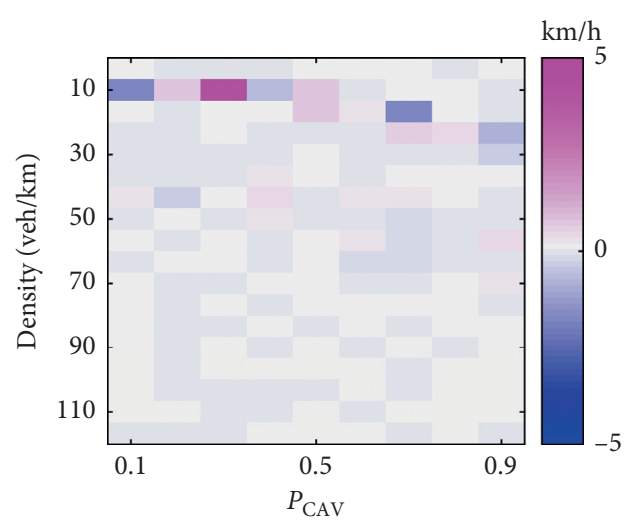

(a)

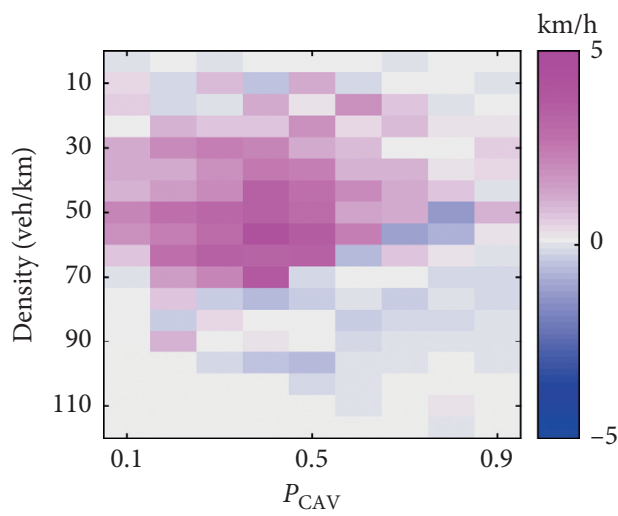

(c)

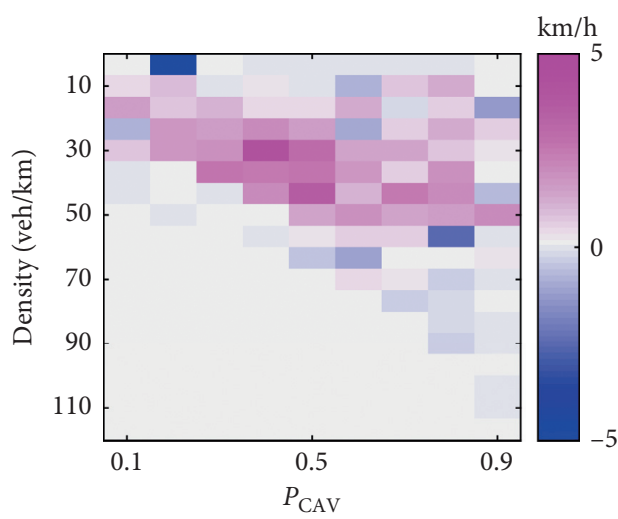

(e)

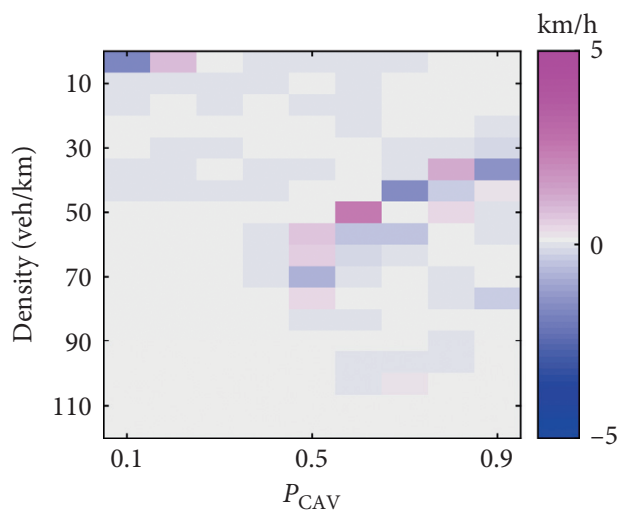

(g)

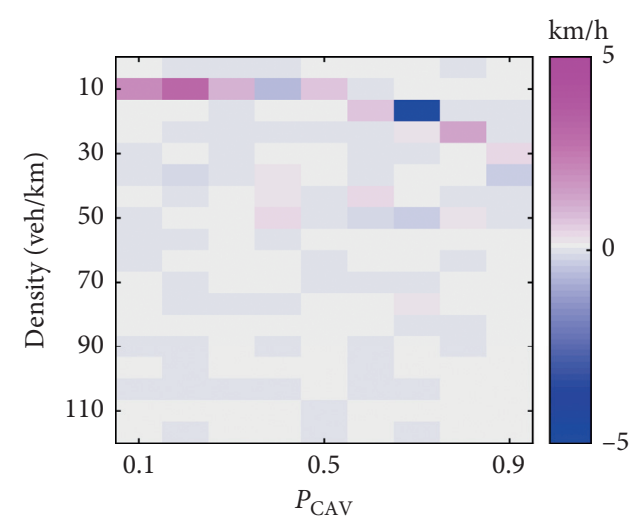

(b)

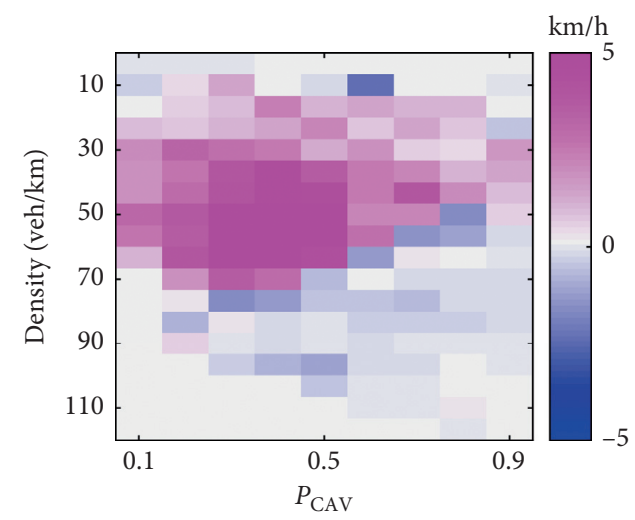

(d)

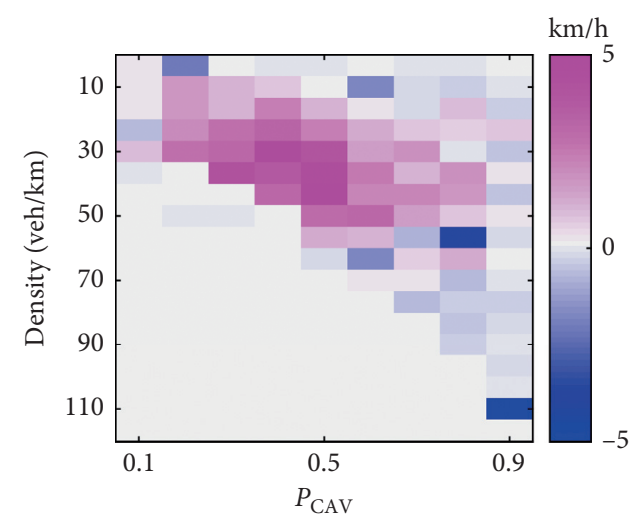

(f)

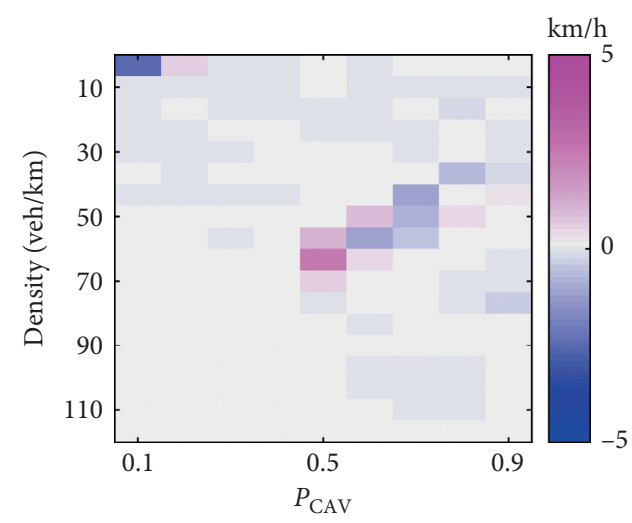

(h)

FIgUre 8: Relationship between speed change and CAV penetration rate with different lane-changing probability of CAVs. (a) $P_{\mathrm{lc}}=0.6$ (GGG). (b) $P_{\mathrm{lc}}=1.0$ (GGG). (c) $P_{\mathrm{lc}}=0.6$ (GCG). (d) $P_{\mathrm{lc}}=1.0$ (GCG). (e) $P_{\mathrm{lc}}=0.6$ (CGC). (f) $P_{\mathrm{lc}}=1.0(\mathrm{CGC})$. (g) $P_{\mathrm{lc}}=0.6(\mathrm{CCM})$. (h) $P_{\mathrm{lc}}=1.0(\mathrm{CCM})$. 


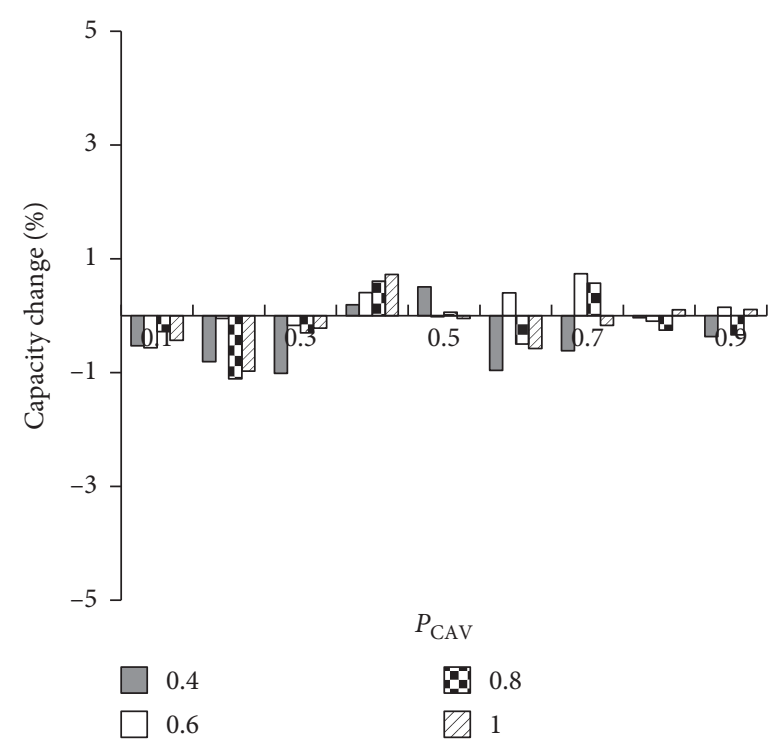

(a)

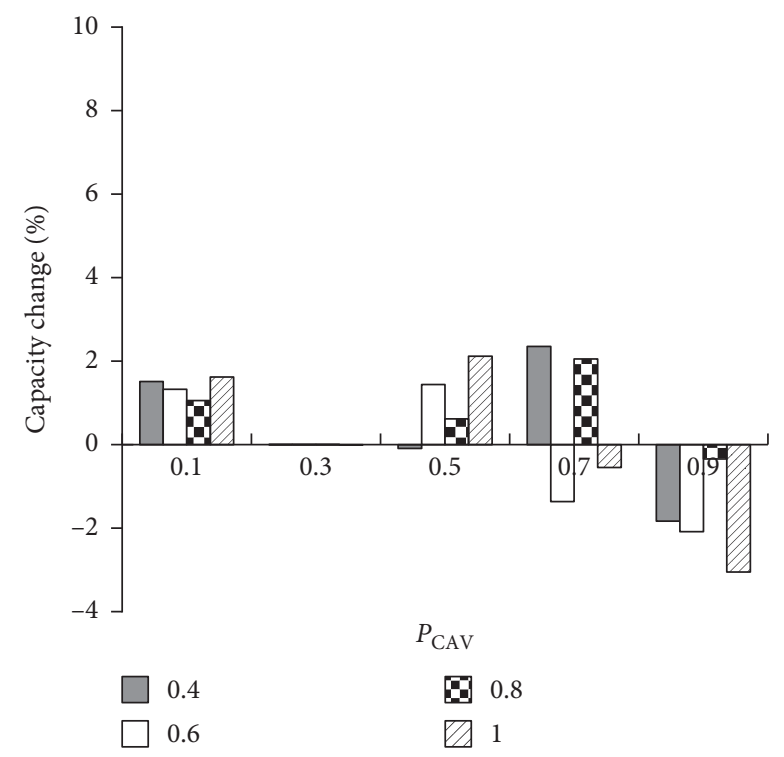

(c)

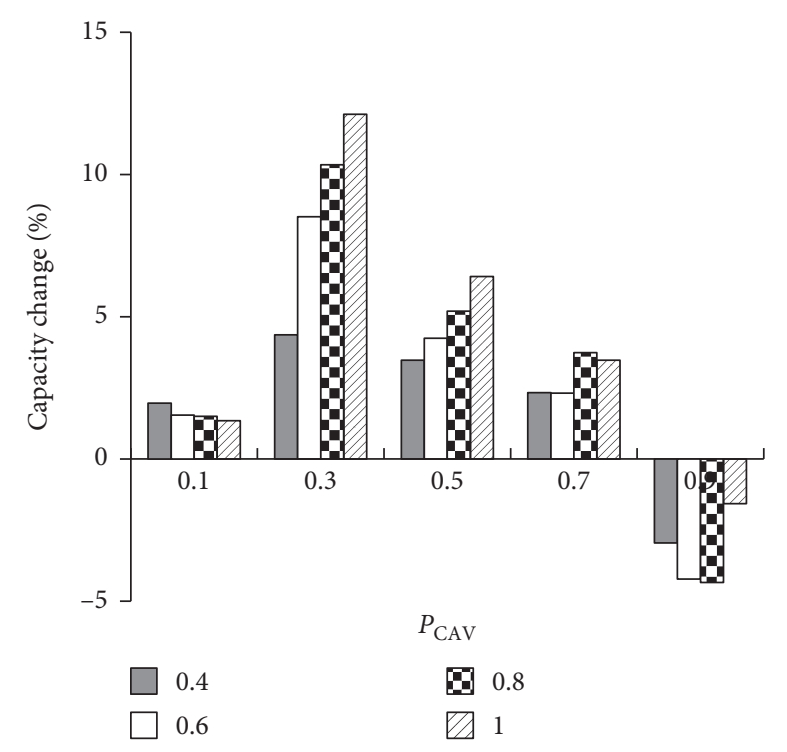

(b)

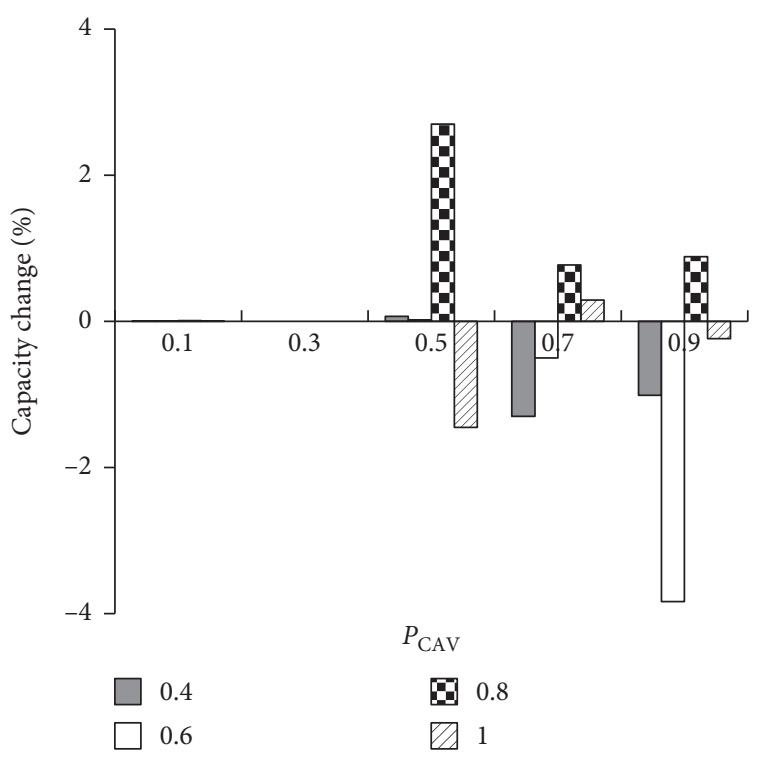

(d)

FIGURE 9: Relationship between capacity change and CAV penetration rate with different lane-changing probabilities of CAVs. (a) GGG. (b) GCG. (c) CGC. (d) CCM.

Figures $8(\mathrm{a}), 8(\mathrm{~b}), 8(\mathrm{~g})$, and $8(\mathrm{~h})$, the influence of lanechanging probability of CAVs on average freeway speed is very slight.

Except for speed, the lane-changing probability may also affect road capacity. Figure 9 illustrates the relationship between capacity change and CAV penetration rate with different lane-changing probabilities of CAVs, when the lane-changing probabilities of CAVs are $0.2,0.4,0.6,0.8$, and 1.0. The capacities when $P_{\mathrm{lc}}=0.2$ are set as benchmarks as well. It is clear that, except GCG policy, the increase of lanechanging probability of CAVs has little impact on road capacity. The capacity improvements are mainly within the range of $-2 \% \sim 2 \%$. When the lane policy is GCG, however, the increase of $\mathrm{P}_{\mathrm{lc}}$ has a positive impact on capacity. In Figure $9(\mathrm{~b})$, when $P_{\mathrm{CAV}}$ is smaller than 0.9. The increase of lane-changing probability of CAVs can enlarge the capacity generally, especially when $P_{\mathrm{CAV}}=0.3$. The largest capacity can be observed when the lane-changing probability of CAVs is 1.0 and $P_{\mathrm{CAV}}=0.3$.

Note that, when CAV penetration rate is over 0.9 , no matter what lane policies are, the increase of lane-changing probability of CAVs will possibly result in a smaller capacity.

\section{Conclusions}

The primary objective of this paper is to study the influence of lane policies on mixed traffic under various traffic volumes and CAV penetration rates. A multilane cellular automaton model was developed, wherein both CAVs and MVs were incorporated in the mixed traffic flow. A 
simulation was then conducted, and various lane policies were considered and compared.

From the simulation results, some major conclusions can be drawn:

(1) The increased number of CAV exclusive lanes can improve the capacity, especially when $P_{\mathrm{CAV}}$ is more than 0.5. MV exclusive lanes and, however, seem helpless for road capacity improvement. In terms of capacity, for two-lane freeways, when $P_{\mathrm{CAV}}$ is no bigger than 0.2 , GG is the best. When $P_{\mathrm{CAV}}$ is over 0.3 , CM policy becomes the best. And, when $P_{\mathrm{CAV}}$ is larger than 0.7, GC policy is recommended. For three-lane freeways, when $P_{\mathrm{CAV}}$ is no less than 0.4 , MMC, GCG, and CGM are recommended, while if $P_{\mathrm{CAV}}$ is over 0.5, CCM, CGC, and CCG are better.

(2) Seven lanes policies, including GC, GM, CM GGG, GCG, CGC, and CCM, perform better in average speed. For two-lane freeways, GM is recommended when $P_{\mathrm{CAV}}$ is less than 0.2 , and $\mathrm{CM}$ and $\mathrm{GC}$ are recommended when $P_{\mathrm{CAV}}$ is more than 0.3 . For three-lane freeways, GGG is recommended when $P_{\mathrm{CAV}}$ is less than 0.3 , and CCM and CGC are recommended when $P_{\mathrm{CAV}}$ is more than 0.4. The advantage of GCG in speed is obvious with high traffic density which is more than $65 \mathrm{veh} / \mathrm{ln} / \mathrm{km}$.

(3) Exclusive lanes can help to reduce the probability that CAVs degenerate into AVs but increase the proportion of no vehicle within CR. The probability CAVs degenerate into AVs reduces with the increase of $P_{\mathrm{CAV}}$ and number of exclusive lanes. CM/CCM can ensure that CAVs do not degenerate into AVs.

(4) Freeways with CAV exclusive lanes are more potential to have a greater average speed gap between CAVs and MVs, showing the superiority of CAVs. This is helpful to increase the penetration rate of CAVs.

(5) A smaller safe headway can increase the road capacity, especially for freeways without exclusive lanes. On the contrary, a larger lane-changing probability of CAVs may be to keep a higher average speed as well as road capacity when the freeway consists of both exclusive lanes and general purpose lanes. One should carefully determine a lanechanging probability and safe headway, to make better use of freeways and lane policies.

Further extension of this paper might consider the influence factors that affect the performance of CAVs, such as time headway, communication delay, and connected range. Freeways with more than three lanes in one direction may have an effect on the road policies, which remains to be studied in the future as well.

\section{Data Availability}

The data used to support the findings of this study are available from the corresponding author upon request.

\section{Conflicts of Interest}

The authors declare that there are no conflicts of interest regarding the publication of this paper.

\section{Acknowledgments}

The research was supported by the National Natural Science of China (71801042 and 51878166) and the Natural Science of Jiangsu Province (BK20180381).

\section{References}

[1] A. Kesting, M. Treiber, and D. Helbing, "Enhanced intelligent driver model to access the impact of driving strategies on traffic capacity," Philosophical Transactions of the Royal Society A: Mathematical, Physical and Engineering Sciences, vol. 368, no. 1928, pp. 4585-4605, 2010.

[2] L. Ye and T. Yamamoto, "Modeling connected and autonomous vehicles in heterogeneous traffic flow," Physica A: Statistical Mechanics and Its Applications, vol. 490, pp. 269277, 2018.

[3] J. Harding, G. Powell, R. Yoon et al., Vehicle-to-Vehicle Communications: Readiness of V2V Technology for Application, National Highway Traffic Safety Administration, Washington, DC, USA, 2014.

[4] J. Santa, A. F. Gómez-Skarmeta, and M. Sánchez-Artigas, "Architecture and evaluation of a unified V2V and V2I communication system based on cellular networks," Computer Communications, vol. 31, no. 12, pp. 2850-2861, 2018.

[5] P. Tientrakool, Y. C. Ho, and N. F. Maxemchuk, "Highway capacity benefits from using vehicle-to-vehicle communication and sensors for collision avoidance," in Proceedings of the 2011 IEEE Vehicular Technology Conference (VTC Fall), pp. 1-5, IEEE, San Francisco, CA, USA, September 2011.

[6] D. Swaroop, J. K. Hedrick, C. C. Chien et al., "A comparision of spacing and headway control laws for automatically controlled vehicles," Vehicle System Dynamics, vol. 23, no. 1, pp. 597-625, 1994.

[7] P. Fernandes and U. Nunes, "Platooning with IVC-enabled autonomous vehicles: strategies to mitigate communication delays, improve safety and traffic flow," IEEE Transactions on Intelligent Transportation Systems, vol. 13, no. 1, pp. 91-106, 2012.

[8] V. Milanés, S. E. Shladover, J. Spring et al., "Cooperative adaptive cruise control in real traffic situations," IEEE Transactions on Intelligent Transportation Systems, vol. 15, no. 1, pp. 296-305, 2014.

[9] A. Ghiasi, O. Hussain, Z. Qian, and X. Li, "A mixed traffic capacity analysis and lane management model for connected automated vehicles: a Markov chain method," Transportation Research Part B: Methodological, vol. 106, pp. 266-292, 2017.

[10] S. Shladover, J. VanderWerf, M. A. Miller et al., Development and Performance Evaluation of AVCSS Deployment Sequences to Advance from Today's Driving Environment to Full Automation, Michigan State University, East Lansing, Michigan, 2001.

[11] D. J. Fagnant and K. Kockelman, "Preparing a nation for autonomous vehicles: opportunities, barriers and policy recommendations," Transportation Research Part A: Policy and Practice, vol. 77, pp. 167-181, 2015. 
[12] H. S. Mahmassani, "Autonomous vehicles and connected vehicle systems: flow and operations considerations," Transportation Science, vol. 50, no. 4, pp. 1140-1162, 2016.

[13] S. E. Shladover, C. A. Desoer, J. K. Hedrick et al., "Automated vehicle control developments in the PATH program," IEEE Transactions on Vehicular Technology, vol. 40, no. 1, pp. 14130, 1991.

[14] P. Ioannou, Z. Xu, S. Eckert, D. Clemons, and T. Sieja, "Intelligent cruise control: theory and experiment," in Proceeding of the 32nd IEEE Conference on Decision and Control, pp. 1885-1890, San Antonio, TX, USA, December 1993.

[15] P. Varaiya, "Smart cars on smart roads: problems of control," IEEE Transactions on Automatic Control, vol. 38, no. 2, pp. 195-207, 1993.

[16] A. Ghiasi, J. Ma, F. Zhou et al., "Speed harmonization algorithm using connected autonomous vehicles," in Proceeding of the 96th Annual Meeting of the Transportation Research Board, Washington, DC, USA, January 2017.

[17] A. Talebpour and H. S. Mahmassani, "Influence of connected and autonomous vehicles on traffic flow stability and throughput," Transportation Research Part C: Emerging Technologies, vol. 71, pp. 143-163, 2016.

[18] S. E. Shladover, D. Su, and X. Y. Lu, "Impacts of cooperative adaptive cruise control on freeway traffic flow," Transportation Research Record, vol. 2324, no. 1, pp. 63-70, 2012.

[19] G. M. Arnaout and J.-P. Arnaout, "Exploring the effects of cooperative adaptive cruise control on highway traffic flow using microscopic traffic simulation," Transportation Planning and Technology, vol. 37, no. 2, pp. 186-199, 2014.

[20] M. Kamrani, B. Wali, and A. J. Khattak, "Can data generated by connected vehicles enhance safety? Proactive approach to intersection safety management," Transportation Research Record, vol. 2659, no. 1, pp. 80-90, 2017.

[21] A. Papadoulis, M. Quddus, and M. Imprialou, "Evaluating the safety impact of connected and autonomous vehicles on motorways," Accident Analysis \& Prevention, vol. 124, pp. 12-22, 2019.

[22] J. Auld, V. Sokolov, and T. S. Stephens, "Analysis of the effects of connected-automated vehicle technologies on travel demand," Transportation Research Record, vol. 2625, no. 1, pp. 1-8, 2017.

[23] S. Learn, J. Ma, K. Raboy, F. Zhou, and Y. Guo, "Freeway speed harmonisation experiment using connected and automated vehicles," IET Intelligent Transport Systems, vol. 12, no. 5, pp. 319-326, 2017.

[24] K. Kwon and P. Varaiya, "Effectiveness of California's high occupancy vehicle (HOV) system," Transportation Research Part C: Emerging Technologies, vol. 16, no. 1, pp. 98-115, 2008.

[25] H. B. Zhu, "Numerical study of urban traffic flow with dedicated bus lane and intermittent bus lane," Physica A: Statistical Mechanics and Its Applications, vol. 389, no. 16, pp. 3134-3139, 2010.

[26] K. Sakamoto, C. Abhayantha, and H. Kubota, "Effectiveness of bus priority lane as countermeasure for congestion," Transportation Research Record, vol. 2034, no. 1, pp. 103-111, 2007.

[27] Z. Chen, F. He, L. Zhang, and Y. Yin, "Optimal deployment of autonomous vehicle lanes with endogenous market penetration," Transportation Research Part C: Emerging Technologies, vol. 72, pp. 143-156, 2016.

[28] S. F. Qom, Y. Xiao, and M. Hadi, "Evaluation of cooperative adaptive cruise control (CACC) vehicles on managed lanes utilizing macroscopic and mesoscopic simulation," in Proceedings of the Transportation Research Board 95th Annual Meeting, Washington, DC, USA, January 2016.
[29] A. Talebpour, H. S. Mahmassani, and A. Elfar, "Investigating the effects of reserved lanes for autonomous vehicles on congestion and travel time reliability," Transportation Research Record, vol. 2622, no. 1, pp. 1-12, 2017.

[30] K. Ma and H. Wang, "Influence of exclusive lanes for connected and autonomous vehicles on freeway traffic flow," IEEE Access, vol. 7, pp. 50168-50178, 2019.

[31] K. Nagel and M. Schreckenberg, "A cellular automaton model for freeway traffic," Journal de physique I, vol. 2, no. 12, pp. 2221-2229, 1992.

[32] B. S. Kerner, "Three-phase traffic theory and highway capacity," Physica A: Statistical Mechanics and Its Applications, vol. 333, pp. 379-440, 2004.

[33] R. Jiang and Q. S. Wu, "The adaptive cruise control vehicles in the cellular automata model," Physics Letters A, vol. 359, no. 2, pp. 99-102, 2006.

[34] J. Tian, G. Li, M. Treiber, R. Jiang, N. Jia, and S. Ma, "Cellular automaton model simulating spatiotemporal patterns, phase transitions and concave growth pattern of oscillations in traffic flow," Transportation Research Part B: Methodological, vol. 93, pp. 560-575, 2016.

[35] M. Treiber and A. Kesting, "Traffic flow dynamics," Traffic Flow Dynamics: Data, Models and Simulation, SpringerVerlag, Berlin Heidelberg, 2013.

[36] B. S. Kerner, "Failure of classical traffic flow theories: stochastic highway capacity and automatic driving," Physica A: Statistical Mechanics and Its Applications, vol. 450, pp. 700747, 2016.

[37] K. Chandan, A. M. Seco, and A. B. Silva, "Real-time traffic signal control for isolated intersection, using car-following logic under connected vehicle environment," Transportation Research Procedia, vol. 25, pp. 1610-1625, 2017. 